\title{
Extended Array Manifolds: Functions of Array Manifolds
}

\author{
Georgios Efstathopoulos, Member, IEEE, and Athanassios Manikas, Senior Member, IEEE
}

\begin{abstract}
The response of an array processing system has been widely modelled using the concept of the array manifold. The conventional array manifold is a function of the geometry of the array, the carrier frequency and the directions of arrival (DOAs) of the sources only. However, the emergence of more sophisticated array systems, for instance antenna array communication systems, dictates the extension of this array manifold into various new types of manifolds that incorporate additional system and channel parameters, such as the code-division multiple access (CDMA) (spreading and scrambling) codes, the lack of synchronization, Doppler effects, polarization parameters, subcarriers, etc. This paper is concerned with the definition and geometric investigation of a generic model for these new manifolds that can be expressed as functions of the conventional array manifolds and, hence, are defined herein as extended array manifolds. Thus, initially, the concept of the extended array manifold is introduced and is shown to accommodate both existing and newly defined extensions of the widely employed in the literature conventional (or spatial) array manifold. Next, the geometric properties and parameters of the extended array manifold are studied and linked with the properties of the associated spatial array manifold in an attempt to facilitate the geometric study of the former by taking advantage of the well understood study of the latter.
\end{abstract}

Index Terms-Array manifold, array processing, differential geometry, extended array manifold.

\section{NOTATION}

$\begin{array}{ll}\text { a } & \text { Scalar. } \\ \underline{a}, \underline{\mathrm{a}} & \text { Column vector. } \\ \text { A } & \text { Matrix. } \\ \underline{0}_{N} & N \text { element column vector of zeros. } \\ (\cdot)^{*},(\cdot)^{T},(\cdot)^{H} & \text { Conjugate, transpose, Hermitian. } \\ \odot, \otimes & \text { Hadamard product, Kronecker product. } \\ \lfloor\cdot\rfloor & \text { Round down to integer. } \\ \|\underline{\mathrm{a}}\| & \text { Euclidean norm of the column vector a. } \\ \underline{a}^{n} & \text { Element-wise exponentiation. }\end{array}$

Manuscript received August 28, 2010; revised January 17, 2011; accepted March 02, 2011. Date of publication March 24, 2011; date of current version June 15,2011 . The associate editor coordinating the review of this manuscript and approving it for publication was Dr. Xavier Mestre.

The authors are with the Department of Electrical and Electronic Engineering, Imperial College London, London, SW7 2AZ, U.K. (e-mail: g.efstathopoulos@gmail.com; a.manikas@imperial.ac.uk).

Digital Object Identifier 10.1109/TSP.2011.2132719

\section{INTRODUCTION}

D IFFERENTIAL geometry is a branch of mathematics which is concerned with the application of differential calculus for the investigation of the properties of curves, surfaces and higher-dimensional mathematical objects having a profound geometrical structure, known as manifolds.

The array response vector a of an array of $N$ omnidirectional sensors (antennas, microphones, etc.) in the presence of a unit-power signal is a mathematical object in a $N$-dimensional complex Hilbert space and is a function of a number of parameters such as the following:

- directions of arrival (DOAs) of the incoming signals (azimuth $\theta$, elevation $\phi$ );

- carrier frequency $F_{c}$;

- velocity of the propagation of the signal $c$;

- positions of the array elements $\underline{r}_{1}, \underline{r}_{2}, \ldots, \underline{r}_{N}$, where $\underline{r}_{i}$ is a $3 \times 1$ real vector with the $x, y, z$ coordinates of the $i$ th sensor.

That is,

$$
\begin{aligned}
& \underline{\mathrm{a}}= \underline{\mathrm{a}}\left(\theta, \phi, F_{c}, \underline{r}_{1}, \underline{r}_{2}, \ldots, \underline{r}_{N}\right) \\
&=\exp \left\{-j \frac{2 \pi F_{c}}{c}\left[\underline{r}_{1}, \underline{r}_{2}, \ldots, \underline{r}_{N}\right]\right. \\
&\left.\cdot[\cos \theta \cos \phi \sin \theta \cos \phi \sin \phi]^{T}\right\} \in \mathcal{C}^{N \times 1}
\end{aligned}
$$

where $\exp \{\underline{a}\}$ denotes element-wise exponentiation of the vector $\underline{a}$.

The locus of the array response vectors for all possible values of the different parameters is the so-called array manifold $\mathcal{A}$, defined formally as

$$
\mathcal{A} \triangleq\{\underline{\mathrm{a}}(\underline{\mathrm{p}}), \underline{\mathrm{p}} \in \Omega\}
$$

where $\mathrm{p}$ is a vector containing all the unknown, variable parameters (which can be any or all of the parameters in the previous list) and $\Omega$ is the parameter space for $\underline{p}$. It is important to note at this point that the array manifold has a profound geometrical structure which as we shall see, greatly facilitates the analysis of array systems.

The array response vector (also known as array manifold vector), as defined in (1), is of limited use in modern communications and remote sensing systems. In these areas, there are additional parameters, apart from the aforementioned ones, which need to be taken into account. Characteristic examples are the pseudo-noise (PN) sequences of spread spectrum systems, subcarriers of multicarrier (MC) systems, polarization 
parameters, Doppler frequency, the lack of synchronization, etc.

Hence, the modelling of such systems requires the introduction of new array response (manifold) vectors, which will depend on additional parameters and may have different dimensions. However, what is important to note is that these new vectors will give rise to new mathematical objects which will have the intrinsic mathematical structure of a manifold as well. More importantly these new manifolds can be expressed, as we shall see shortly, as functions of the array manifold of (2) and for this reason will be referred to, henceforth, as extended array manifolds. For the remaining of this paper the symbols $\underline{h}(\underline{\mathrm{p}})$ and $\mathcal{H}$ will be used to denote an extended array manifold vector and the associated extended array manifold defined as

$$
\mathcal{H} \triangleq\{\underline{h}(\underline{\mathrm{p}}), \underline{\mathrm{p}} \in \Omega\}
$$

where, again, $p$ is a vector containing all the unknown, variable parameters and $\Omega$ is the parameter space for $\mathrm{p}$.

The extended array manifolds depend on various parameters, including of course all those on which the array manifold depends and have been presented in the beginning of this paper. Some of these are system parameters and if the array is calibrated they are usually known and fixed. We will assume in this work that this is the case. Examples of such known and fixed parameters are the carrier frequency $F_{c}$ and the locations of the array sensors $\underline{r}_{i}, i=1, \ldots, N$. The remaining will be channel parameters, such as the DOAs of the incoming signals and the Doppler spread. These are considered unknown and sought after parameters. In this paper we will only consider the cases where we have either one or two unknown parameters.

If there is only one unknown parameter $p$, then the array manifold vector $\underline{\mathrm{a}}(\underline{\mathrm{p}})$ and any extended array manifold vector $\underline{h}(\underline{\mathrm{p}})$ become

$$
\underline{\mathrm{a}}=\underline{\mathrm{a}}(p) \in \mathcal{C}^{N} \text { and } \underline{h}=\underline{h}(p) \in \mathcal{C}^{Q}
$$

and the associated manifolds $\mathcal{A}$ and $\mathcal{H}$ represent curves embedded in multidimensional complex spaces.

If there are two unknown parameters $p$ and $q$, then we have

$$
\underline{\mathrm{a}}=\underline{\mathrm{a}}(p, q) \in \mathcal{C}^{N} \text { and } \underline{h}=\underline{h}(p, q) \in \mathcal{C}^{Q}
$$

and the resulting manifolds $\mathcal{A}$ and $\mathcal{H}$ represent surfaces embedded in multidimensional complex spaces.

\section{A. The Geometry of the Array Manifold}

The (spatial or conventional) array manifold $\mathcal{A}$ of an array of $N$ sensors is a multidimensional complex nonlinear subspace in $\mathcal{C}^{N}$ (or equivalently in $\mathcal{R}^{2 N}$ ) equipped with the standard complex structure [1]. In Differential Geometry, this nonlinear subspace $\mathcal{A}$ is a mathematical object (i.e., curve, surface, etc.) embedded in $\mathcal{C}^{N}$. Differential Geometry is a branch of mathematics that is concerned with the application of differential calculus for the investigation of the properties of geometric space curves, surfaces and other mathematical objects known as "manifolds" in multidimensional spaces. These mathematical objects (manifolds) have a deep and profound mathematical structure and have been used to express many significant problems in physics.

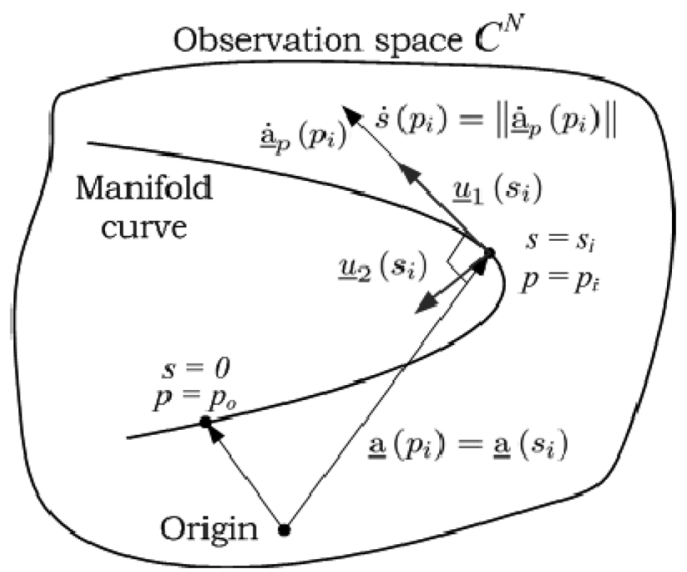

Fig. 1. Graphical representation of the array manifold curve in $\mathcal{C}^{N}$. The parameters $\underline{u}_{1}$ and $\underline{u}_{2}$ are the first two coordinate vectors, $p$ and $s$ are the directional and natural parameter respectively, $\dot{s}(p)$ is the rate of change of the arc length and $\underline{\dot{a}}(p)$ is the tangent vector to the array manifold curve.

In array processing, differential geometry has been used to study the conventional array manifold $\mathcal{A}$ and for this reason it is useful at this point to review some of the most important results of the geometrical investigation of the conventional array manifold $\mathcal{A}$.

1) Array Manifold Curves: If the array manifold is a function of one parameter only, it corresponds to a curve embedded in a complex $N$-dimensional space and (2) becomes

$$
\mathcal{A}=\left\{\underline{\mathrm{a}}(p) \in \mathcal{C}^{N}, \forall p: p \in \Omega_{p}\right\}
$$

where $\Omega_{p}$ is the parameter space for $p$ and $p$ may be the azimuth angle $\theta$, the elevation angle $\phi$ or any other equivalent channel parameter of interest that, however, has to be related to the free variable $(\theta$ or $\phi)$ via an allowable change of parameters. The geometric properties of array manifold curves have been extensively studied in the literature [2]-[4].

The most important parameters of the array manifold curve (see Fig. 1 for a graphical representation) at some point $p$ are as follows:

- the tangent vector $\underline{\dot{a}}(p) \triangleq \frac{\partial \mathrm{a}}{\partial p}$;

- the arc length $s(p)$;

- the rate of change of the arc length $\dot{s}(p)$;

- the set of coordinate vectors $\underline{u}_{i}(p), i=1, \ldots, d$ at a point $p$ of the manifold curve;

- the set of curvatures of the manifold curve $\kappa_{i}(p), i=$ $1, \ldots, d$, which form the Cartan matrix $\mathbb{C}(p)$

$$
\mathbb{C}(p)=\left[\begin{array}{cccccc}
0 & -\kappa_{1} & 0 & \ldots & 0 & 0 \\
\kappa_{1} & 0 & -\kappa_{2} & \ldots & 0 & 0 \\
0 & \kappa_{2} & 0 & \ldots & 0 & 0 \\
\vdots & \vdots & \vdots & \ddots & \vdots & \vdots \\
0 & 0 & 0 & \ldots & 0 & -\kappa_{d-1} \\
0 & 0 & 0 & \ldots & \kappa_{d-1} & 0
\end{array}\right] .
$$

The arc length $s(p)$ of the array manifold curve is formally defined as

$$
s(p)=\int_{p_{o}}^{p}\|\underline{\dot{a}}(p)\| d p .
$$


The arc length $s(p)$ is an invariant parameter, that is the tangent vector to the curve, expressed in terms of $s, \underline{\mathrm{a}}^{\prime}(s) \triangleq \frac{d \underline{\mathrm{a}}(s)}{d s}$, has always unity norm [5].

The rate of change of the manifold length

$$
\dot{s}(p)=\|\underline{\dot{a}}(p)\|
$$

is a local property of the curve and is directly related to the resolution and detection capabilities of the array [6].

At any point $\underline{\mathrm{a}}(p)$ of a curve embedded in $\mathcal{C}^{N}$, it is possible to attach a continuous, differentiable and orthonormal, in the "wide" sense, ${ }^{1}$ system of $2 N$ coordinate vectors $\underline{u}_{i}(p), i=$ $1, \ldots, 2 N$ [3], [7]. These coordinate vectors are indispensable in the differential geometric treatment of curves as it is easier to describe local properties (e.g., curvature, torsion) in terms of a local reference system than a global one, like an orthonormal Euclidean coordinate system attached at the origin.

According to the fundamental uniqueness theorem [7] curvatures (and thus the Cartan matrix $\mathbb{C}$ ) uniquely define a space curve, except for its position in space. Moreover, the number of nonzero curvatures reveal symmetries in the geometry of the array manifold curve, since a curve with $d-1, d \leq 2 N$ nonzero curvatures is restricted to a $d$-dimensional subspace of $\mathcal{R}^{2 N}$. It can be proven that if the curvatures of the array manifold remain constant, then the shape of $\mathcal{A}$ will be a hyperhelix. This property of manifold curves is of high importance, since hyperhelical curves are easy to study and analyze due to their specific geometric structure [3].

2) Array Manifold Surfaces: Array manifolds that are functions of two free variables, e.g., $(p, q)$, correspond to surfaces $\mathcal{M}$ embedded in $\mathcal{C}^{N}$. The most important geometric properties of space surfaces embedded in multidimensional complex spaces are the following [3], [8]:

- the manifold metric $\mathbb{G}(p, q)$;

- the Christoffel matrices $\Gamma_{1}(p, q)$ and $\boldsymbol{\Gamma}_{2}(p, q)$;

- the Gaussian curvature $K_{G}(p, q)$;

- the geodesic curvature $\kappa_{g}$ of a curve on $\mathcal{M}$.

Their physical interpretation is described below.

The manifold metric

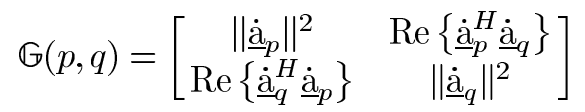

is a $2 \times 2$ semi-positive definite symmetric matrix, with its elements known, in differential geometry terms, as the first fundamental coefficients (or metric coefficients). It is a function of the tangent vectors $\underline{\dot{a}}_{p}=\frac{\partial \underline{\mathrm{a}}(p, q)}{\partial p}$ and $\underline{\dot{\mathrm{a}}}_{q}=\frac{\partial \underline{\mathrm{a}(p, q)}}{\partial q}$, which form a basis

$$
\mathbb{T}=\left[\underline{\dot{a}}_{p}, \underline{\dot{a}}_{q}\right]
$$

of the tangent plane of the surface at the point $(p, q)$. The first fundamental coefficients provide a way of measuring trajectories on non-Euclidean spaces. Furthermore, $\sqrt{\operatorname{det}(\mathbb{G})}$ serves as

${ }^{1}$ Wide sense means $\operatorname{Re}\left\{\underline{u}_{i}^{H} \underline{u}_{j}\right\}=\delta_{i j}$. a tool for detecting the changes of the shape of the manifold surface [5], while the area of a segment on the manifold surface can be calculated as $\iint_{w} \sqrt{\operatorname{det}(\mathbb{G})} d p d q$.

The Christoffel symbol matrices of the first kind $\Gamma_{1}$ and second kind $\boldsymbol{\Gamma}_{2}$ determine how the tangent plane varies as the point $(p, q)$ moves on the manifold surface.

According to the Theorema Egregium by Gauss, to every point of a surface embedded in $\mathcal{R}^{3}$ can be assigned a real number $K_{g}$, independently of any specific curve passing through it. This real number, the Gaussian curvature, provides an indication of the local shape of the surface at the neighborhood of that point. In [3], the aforementioned theorem has been extended to manifold surfaces embedded in a complex $N$-dimensional space, where the definition of the Gaussian curvature utilizes the intrinsic geometry of the manifold surface.

Finally, for curves lying on manifold surfaces two important parameters are the arc length $s$ and the geodesic curvature $\kappa_{g}$. The arc length of a curve has already been defined in the previous paragraph. The geodesic curvature $\kappa_{g}$ is defined using the first curvature $\kappa_{1}$ of the curve along the tangent plane to the surface at every point along the curve. By considering a curve on a surface connecting two points, the geodesic curvature assesses the similarity of this curve to a geodesic curve (i.e., a curve which is equivalent to a straight line in Euclidean space).

3) Array Manifold Surfaces as Family of Curves: The investigation of a surface $\mathcal{M}$ as a mathematical object embedded in $N$-dimensional complex space is more complex than that of curves. Thus, an alternative representation using two families of parameter curves has been proposed in [3] to express $\mathcal{M}$. These are the family of $p$-curves defined as

$$
\left\{\mathcal{A}_{p \mid q_{o}}, \forall q_{o}: q_{o} \in \Omega_{q}\right\}
$$

and the family of $q$-curves defined in a similar fashion. For a given (fixed) parameter $q_{o}$, the curve $\mathcal{A}_{p \mid q_{o}}$ is defined as a $p$-curve and the ensemble of $p$-curves provide a covering for the whole manifold surface. By treating the manifold surface using the aforementioned two families of curves, then a unified framework for the analysis of linear and nonlinear array geometries is possible.

\section{B. Exemplary Results and Motivation}

Studying the array manifold as a mathematical object embedded in a proper multidimensional complex space has led to a number of significant results regarding the analysis of arraybased systems. The problem of ambiguities in array processing has been analyzed and linked with array manifold geometric properties in [9]. In [10], theoretical lower bounds regarding the detection, resolution and accuracy capabilities of linear and some planar array systems have been derived as function of the intrinsic geometry of the array manifold. The authors in [11] utilize differential geometry to determine the Cramér-Rao bound for arrays composed of widely separated subarrays. In [4] geometric concepts have been utilized to tackle the array design problem in an optimal way. Geometric information about the array manifold was also used in [12], where the author utilised the array manifold geometry to perform array design under accuracy and peak side-lobe level constraints and in [13] for array 
manifold interpolation. In addition, in [14] the authors study the array geometries which give rise to isotropic array performance with regards to the signal DOA. Finally, in [15] study the effect of array geometry in DoA estimation accuracy and array ambiguity and utilize the derived results to perform array design.

These promising results have motivated the introduction of new array manifold models, such as a generalized array manifold for the modelling of local scatterers in [16] and an array manifold for acoustic vector-sensor (i.e., pressure and velocity measurements) array in [17]. In addition, is a series of papers [18]-[20], the authors proposed a new approach to representing and simplifying the analysis of the array manifold.

Some exemplary results with very significant consequences to array processing as well as multiantenna wireless systems (e.g., SIMO, MISO, MIMO) are summarized below [3].

- Any linear array of sensors/antennas with a uniform or nonuniform, symmetric, or asymmetric geometry can be expressed as a curve having a "hyperhelical" shape embedded in a multidimensional complex space. "Hyperhelices" are very important and useful space curves as for each hyperhelix there is a set of constant numbers (known as curvatures) that fully describe the curve and consequently the array of sensors and its performance.

- The manifolds of 3D-grid arrays (for example a cube of $N=8$ antennas) can be expressed as a surface of spherical shape, embedded in $\mathcal{C}^{N}$.

- All planar arrays have a manifold surface of conoidal shape.

- If $(p, q)$ represent the azimuth and elevation angles respectively, then manifold surfaces of 2- and 3-dimensional arrays can be expressed as families of azimuth curves and elevation curves. For the case of 2-dimensional arrays, elevation curves are hyperhelices while azimuth curves are not.

- For planar arrays, if $(p, q)$ represent the cone angles $(\alpha, \beta)$ then we have two families of hypehelical curves describing the manifold surface.

However, the emergence of more sophisticated array systems, for instance wireless communication systems that employ an antenna array of a given geometry, dictates the extension of its array manifold into various new types of manifolds that incorporate additional system and channel parameters, such as the CDMA (spreading and scrambling) codes, the lack of synchronization, Doppler effects, polarization parameters, subcarriers etc.

While the study of systems using differential geometry is well understood and straightforward for spatial/conventional manifolds, this is not the case if additional system/channel parameters are incorporated giving rise to "new" manifolds. The work presented in this paper is an initial attempt that is concerned with the definition and investigation of a generic model for these new manifolds using differential geometry. As these new manifolds can be expressed as functions of the conventional array manifolds, are defined herein as "extended" array manifolds.

Although the theoretical geometric investigation presented in this paper is not connected to any specific application, it is important to point out that the significance and power of differential geometry in array processing and in wireless systems that employ an antenna array is not to replace but to complement the statistical array processing and Shannon's communication theory aiming at the following:

- expressing an array processing system, or other wireless systems, as a space curve, a surface or other mathematical objects;

- analyzing or designing such systems by analyzing or designing curves or surfaces;

- providing, in conjunction with the statistical array processing and information theory, new, more powerful algorithms/systems and open up new applications.

\section{Paper Organization}

Initially, in Section II, some representative examples of extended array manifold vectors that incorporate new parameters from wireless communication systems that employ an array of $N$ antennas are presented-expressed as functions of the conventional array manifold vector. Then the concept of the extended array manifold $\mathrm{H}$ is formally defined. In Sections III and IV, the geometric properties of the extended array manifold curves and surfaces, respectively, are investigated and derived as functions of the corresponding properties of the array manifold curves and surfaces - in an attempt to facilitate the differential geometric analysis of the former by taking advantage of the well understood properties of the latter. Finally, the paper is concluded in Section V.

\section{EXTENSIONS OF THE ARRAY MANIFOLD}

\section{A. Examples of Extended Array Manifolds}

Some examples of extensions of the array manifold vector of (1), which have been introduced in the literature so far, will now be given. These examples will not only provide us with some insight into the nature of these extensions, but will also motivate our analysis of the geometric properties of extended array manifolds.

In [21], an asynchronous, CDMA array system has been modeled using the STAR (Spatio-Temporal ARray) manifold vector

$$
\underline{h}^{\mathrm{STAR}}(p, q, l)=\underline{\mathrm{a}}(p, q) \otimes\left(\mathcal{J}_{2 N_{c}}^{l} \underline{c}\right) .
$$

This new manifold vector is a function of the geometry of the array and the directional parameters of the signal $p$ and $q$ (included in $\underline{a}(p, q))$, the PN sequence of length $N_{c}$ of each user contained in the $2 N_{c}$ vector $\underline{c}$ (which is padded with $N_{c}$ zeros at the end) and the lack of synchronization

$$
l=\left\lceil\frac{\tau_{i j}}{T_{c}}\right\rceil \bmod N_{c}
$$

where $l$ is the discretized delay $\tau_{i j}$ of the $j$ th path of the $i$ th user in units of the code chip period $T_{c}$. Finally,

$$
\sqrt{2}_{2 N_{c}}=\left[\begin{array}{cc}
\underline{0}_{2 N_{c}-1}^{T} & 0 \\
\mathbb{\beth}_{2 N_{c}-1} & \underline{0}_{2 N_{c}-1}
\end{array}\right]
$$

is the downshifting matrix of size $2 N_{c} \times 2 N_{c}$. Note that henceforth $\rrbracket_{K}$ will denote the $K \times K$ downshifting matrix.

Multi-carrier CDMA systems are strong candidates for the next generation of wireless communications, because of their inherent ability to tackle frequency selective channels, while at 
the same time enjoying the advantages of spread-spectrum systems. An array system using multicarrier transmission is introduced in [22] and modelled with the use of the MC-STAR manifold vector

$$
\underline{h}^{\mathrm{MC}-\operatorname{STAR}}\left(p, q, l, F_{k}\right)=\underline{\mathrm{a}}(p, q) \otimes\left(\mathcal{J}_{2 N_{c} N_{s c}}^{l}\left[l, F_{k}\right]\right)
$$

where

$$
\underline{a}\left[l, F_{k}\right] \triangleq \gamma[k]\left[\begin{array}{c}
c[0] e^{j 2 \pi F_{k}(-l) T_{s}} \\
c\left[\left\lfloor\frac{1}{N_{s c}}\right\rfloor\right] e^{j 2 \pi F_{k}(1-l) T_{s}} \\
\vdots \\
c\left[\left\lfloor\frac{2 N_{c} N_{s c}-1}{N_{s c}}\right\rfloor\right] e^{j 2 \pi F_{k}(L-1-l) T_{s}} \\
\underline{0}_{N_{c} N_{s c}}
\end{array}\right],
$$

$N_{s c}$ is the number of different subcarriers used in the multicarrier modulation and $\underline{\gamma}=\left[\gamma[1], \ldots, \gamma\left[N_{s c}\right]\right] \in \mathcal{Z}^{N_{s c}}$ is a second $\mathrm{PN}$-sequence used to spread the data among the subcarriers. In addition, $\gamma[k]$ and $F_{k}$ are the PN-chip and the subcarrier frequency corresponding to the $k$ th subcarrier.

Polarization information has been used in the literature to improve the detection and estimation capabilities of the array [23] and to deal with correlated [24] or coherent [25] sources. In order to model the effect of the signal polarization on the array system, the Polar-STAR manifold vector $\underline{h}^{\text {POL-STAR }}$ was introduced in [26]:

$$
\underline{h}^{\mathrm{POL}-\operatorname{STAR}}(p, q, l, \gamma, \eta)=\underline{\mathrm{a}}(p, q) \otimes \underline{\mathrm{q}}(p, q, \gamma, \eta) \otimes\left(\mathbb{J}_{2 N_{c}}^{l} \underline{c}\right)
$$

where $q$ (see [23]) models the effect of a signal with a state of polarization $(\gamma, \eta)$.

Finally, in [27] the authors presented an array system affected by Doppler effect and defined the Doppler-STAR manifold vector

$$
\underline{h}^{\mathrm{DOP}-\operatorname{STAR}}\left(p, q, l, f_{D}, n\right)=\underline{\mathrm{a}}(p, q) \otimes\left(\boldsymbol{l}_{2 N_{c}}^{l} \underline{c} \odot \underline{\mathcal{F}}_{D}[n]\right)
$$

where

$$
\underline{\mathcal{F}}_{D}[n]=\left[\begin{array}{c}
1 \\
\exp \left(j 2 \pi f_{D} T_{c}\right) \\
\vdots \\
\exp \left(j 2\left(2 N_{c}-1\right) \pi f_{D} T_{c}\right)
\end{array}\right] \exp \left\{j 2 \pi n f_{D} T_{c s}\right\}
$$

and $f_{D}$ is the Doppler spread affecting the signal. Notice that the nonstationarity of the channel is reflected on the dependence of the Doppler-STAR manifold vector on the time index $n$.

\section{B. The Extended Manifolds as Complex Mappings}

All of the extended array manifold vectors presented in Section II-A are functions of the array manifold vector $\underline{\mathrm{a}}=\underline{\mathrm{a}}(p, q)$. In detail, for reasons that will be made clear in this section, any extended array manifold can be viewed as the result of a complex mapping, satisfying certain constraints and acting on the array manifold. The nature of these constraints, as well as the general properties of these mappings will be investigated next.

Let us consider an injective, analytic complex mapping $\mathcal{T}$ : $\mathcal{C}^{N} \mapsto \mathcal{C}^{Q}$. An "analytic mapping" $\mathcal{T}$ is a vector function, every component of which, $\mathbf{f}_{q}: \mathcal{C}^{N} \mapsto \mathcal{C}, q=1, \ldots, Q$, is an analytic function. Applied on the array manifold vector $\underline{a}$, this mapping produces a new vector $\underline{h} \in \mathcal{C}^{Q}, Q \geq N$, that is

$$
\underline{h}=\mathcal{T}(\underline{\mathrm{a}})=\left[\mathbf{f}_{1}(\underline{\mathrm{a}}), \mathbf{f}_{2}(\underline{\mathrm{a}}), \ldots, \mathbf{f}_{Q}(\underline{\mathrm{a}})\right]^{T} .
$$

$\mathcal{T}$ maps the manifold vector $\underline{\mathrm{a}}(p, q)$ to the extended array manifold $\underline{h}(\mathrm{p})$. Because $\mathcal{T}$ is both analytic (therefore continuous) and injective, it is an embedding [5]. Therefore, since the conventional array manifold of (2) is a manifold, applying the embedding $\mathcal{T}$ will produce a new manifold.

The extended array manifold will consist of, in general, $Q$ analytic functions $\mathbf{f}_{q}, q=1, \ldots, Q$ of the array manifold vector. However, herein, we will focus on specific linear mappings $\mathcal{T}$, so that (19) can be rewritten as

$$
\underline{h}(\underline{\mathrm{p}})=\mathrm{A}(\underline{\mathrm{p}}) \underline{\mathrm{a}}(p, q)
$$

where $A(\underline{p})$ is a matrix of orthogonal columns, i.e.,

$$
\mathbb{A}^{H} \mathbb{A} \in \mathcal{R}^{N \times N} \text { and is diagonal }
$$

and depends on the specific array system under examination. It is important to point out that the majority of the examined extended array manifolds have a matrix $A(p)$ of the following form:

$$
A=\rrbracket_{N} \otimes \underline{z}(\underline{\mathrm{p}})
$$

where

$$
\underline{z}(\underline{\mathrm{p}}) \in \mathcal{C}^{\frac{Q}{N}}, \quad \frac{Q}{N} \in \mathcal{N}
$$

with the vector $\underline{z}(\underline{p})$ depending again on the specific array system under examination. For instance, in the case of the STAR and the MC-STAR manifold vectors of (13) and (16),

$$
\begin{aligned}
\underline{z}_{\operatorname{STAR}}(\underline{\mathrm{p}}) & =\mathbb{J}_{2 N_{c}}^{l} \underline{c} \\
\underline{z}_{\mathrm{MC}-\operatorname{STAR}}(\underline{\mathrm{p}}) & =\mathbb{J}_{2 N_{c} N_{s c}}^{l}\left[l, F_{k}\right] .
\end{aligned}
$$

Finally, note that (22) satisfies the conditions of (21).

\section{EXTENDED MANIFOLD CURVES}

\section{A. Basic Theoretical Framework}

Like the conventional array manifold, the extended array manifolds are also mathematical objects embedded in multidimensional complex spaces. In this section, manifolds which depend on one parameter only (i.e., curves) will be considered. Since the investigation of the array manifold $\mathcal{A}$ has focused on the bearing curves (i.e., azimuth, elevation, directional cosine curves), the parameter $p$ in the following analysis will stand, as before, for a directional parameter, which implies that the vector parameter $\mathrm{p}$ reduces to the scalar parameter $p$. In this case, the locus of all extended manifold vectors $\underline{h}(p)$ defined by (20) is given by

$$
\mathcal{H}_{c} \triangleq\left\{\underline{h}(p) \in \mathcal{C}^{Q}, \forall p: p \in \Omega_{p}\right\}
$$

where $\Omega_{p}$ is the parameter space for $p$. Equation (26) defines a curve embedded in a $Q$-dimensional complex space. 
The tangent vector at each point $p$ of the extended manifold curve is given by

$$
\underline{\dot{h}}(p) \triangleq \frac{\partial \underline{h}(p)}{\partial p}=\frac{\partial \mathrm{A}(p)}{\partial p} \underline{\mathrm{a}}(p)+\mathrm{A}(p) \frac{\partial \underline{\mathrm{a}}(p)}{\partial p} .
$$

For the sake of simplicity, the dependence of the various vectors and matrices on the variable $p$ will be dropped and only explicitly mentioned when it is needed. Moreover, $\dot{x}$ will denote differentiation of $x$ with regards to the variable $p$.

The rate of change of the arc length of $\mathcal{H}_{c}$ is

$$
\begin{aligned}
& \dot{s}_{\mathcal{H}_{c}}(p)=\|\underline{\underline{h}}(p)\| \\
& =\sqrt{\underline{\dot{a}}^{H} A^{H} A \underline{\dot{a}}+\underline{a}^{H} \dot{\mathrm{A}}{ }^{H} \dot{\AA} \underline{a}+2 \operatorname{Re}\left\{\underline{a}^{H} \dot{\mathrm{A}}^{H} \AA \underline{\dot{a}}\right\}} \text {. }
\end{aligned}
$$

However

$$
\mathbb{A}^{H} \mathbb{A}=\underbrace{\|\underline{z}(p)\|^{2}}_{\triangleq \sigma_{\dot{A}}^{2}(p)} \rrbracket_{N}, \quad \dot{A}^{H} \dot{A}=\underbrace{\|\underline{\dot{z}}(p)\|^{2}}_{\triangleq \sigma_{\dot{A}}^{2}(p)} \rrbracket_{N}
$$

and $\underline{a}^{H} \underline{\dot{a}}=0$ because of the fact that the array centroid has been taken as the array reference point (i.e., $\underline{1}_{N}^{T} \underline{r}_{x}=\underline{1}_{N}^{T} \underline{r}_{y}=$ $\underline{1}_{N}^{T} \underline{r}_{z}=0$ ). Note this condition is valid for any array geometry, since we can always choose a suitable origin for the coordinate system. Based on (29), (28) may be rewritten as

$$
\dot{s}_{\mathcal{H}_{c}}(p)=\sqrt{\sigma_{\text {A }}^{2}(p) \dot{s}_{\mathcal{A}}^{2}(p)+\sigma_{\dot{\mathrm{A}}}^{2}(p) N}
$$

where

$$
\dot{s}_{\mathcal{A}}(p) \triangleq \sqrt{\underline{\dot{\hat{\mathrm{a}}}}^{H} \underline{\dot{\mathrm{a}}}}=\pi \sin p\left\|\underline{r}_{x}\right\|
$$

is the rate of change of arc length of the corresponding array manifold curve $\mathcal{A}$ (see (6)). It is important to note that the rate of change of arc length of the extended manifold curve $\mathcal{H}_{c}$ has been expressed as a function of the corresponding geometric property of the array manifold curve $\mathcal{A}_{c}$ and the scalar functions $\sigma_{\text {A }}(p)$ and $\sigma_{\dot{A}}(p)$ which code the properties of the mapping $\mathcal{T}$.

Equation (30) provides a deeper insight into the relation between the two manifold curves. The right-hand side of (30) consists of two terms; the first one of these terms is the square of rate of change of arc length of the manifold curve $\mathcal{A}$ scaled by the factor $\sigma_{\AA}^{2}(p)$. The second term includes the factor $\|\underline{\underline{z}}(p)\|^{2}$ which can be regarded as the rate of change of the arc length of a new manifold curve $\mathcal{A}_{\underline{z}}$, defined as

$$
\mathcal{A}_{\underline{z}} \triangleq\left\{\underline{z}(p) \in \mathcal{C}^{\frac{Q}{N}}, \forall p: p \in \Omega_{p}\right\} .
$$

The multiplicative factors $\sigma_{\mathrm{A}}^{2}(p)$ and $N$ act as weighting factors because of the different dimensionality of the embedding spaces for the two curves. The total length of the curve $\mathcal{H}_{c}$ is given by

$$
\ell_{\mathcal{H}_{c}}=\int_{\Omega_{p}} \dot{s}_{\mathcal{H}_{c}}(p) \mathrm{d} p
$$

where $\Omega_{p}$ is the domain of $p$. The first coordinate vector, a unit vector co-linear with the tangent vector $\underline{\dot{h}}(p)$ can be expressed as

$$
\underline{u}_{1}=\underline{u}_{1}(s) \triangleq \frac{\mathrm{d} \underline{h}}{\mathrm{~d} s}=\frac{\underline{\dot{h}}(p)}{\dot{\boldsymbol{s}}_{\mathcal{H}_{c}}(p)}
$$

where $s$ is the total arc length of the manifold curve up to the point $p$ and it can be related to the bearing parameter $p$ via the allowable change of parameter [5] defined in

$$
s_{\mathcal{H}_{c}}(p)=\int_{p_{o}}^{p} \dot{s}_{\mathcal{H}_{c}}(p) \mathrm{d} p .
$$

Because the extended manifold curves are embedded in a $Q$-dimensional complex space, it is possible to attach to every point of the manifold curve up to $2 Q$ coordinate vectors and $2 Q$ curvatures, which form the Cartan matrix $\mathbb{C}$ and uniquely define the shape of the manifold curve [5], [7]. However, any symmetry in the geometry of the array system, i.e., any symmetry in the positions of the array elements, will reduce the dimensionality of the subspace in $\mathcal{C}^{Q}$ in which the array manifold curve is embedded [3]. How this reduction takes place is out of the scope of this paper. However, in general up to $d$ coordinate vectors may be defined, where $\frac{d}{2}$ is the dimensionality of the complex subspace in $\mathcal{C}^{Q}$ in which the array manifold curve lies.

These $d$ coordinate vectors and curvatures can be analytically calculated by applying the formulae presented in [3] to the model of the extended array manifolds presented earlier in this paper and making use of (26)-(31). The derivation of all the coordinate vectors and subsequently the curvatures of the extended manifold curves, although analytically possible, is in practise extremely arduous. However, the most interesting differential geometric properties from an array processing point of view are as follows:

- the first and second coordinate vectors $\underline{u}_{1}, \underline{u}_{2}$;

- the rate of change of the arc length $\dot{s}(p) \triangleq \frac{\mathrm{d} s}{\mathrm{~d} p}$;

- and the first curvature $\kappa_{1}$.

These properties have been used extensively in the study of array systems, such as in determining the resolution and detection capabilities of linear [10] and 3-D [6] array geometries, the Cramér-Rao Lower bound for the estimation of channel parameters [28], analyzing the ambiguities of linear and planar arrays [9] and finally in array design [29].

The second coordinate vector $\underline{u}_{2}(s)$ and the first curvature $\kappa_{1}$ can be calculated using the following equations:

$$
\begin{aligned}
& \underline{u}_{2}(s)=\frac{\underline{u}_{1}^{\prime}(s)}{\left\|\underline{u}_{1}^{\prime}(s)\right\|} \\
& \kappa_{1}(s)=\left\|\underline{u}_{1}^{\prime}(s)\right\|
\end{aligned}
$$

where $x^{\prime}$ denotes differentiation with regards to the natural parameter $s$ (arclength). Based on these definitions and on (27)-(34) it is possible to show that the principal (first) curvature and the second coordinate vector are

$$
\begin{aligned}
& \kappa_{1}(p)=\frac{\left\|\ddot{\underline{\hat{H}}}(p) \dot{\dot{s}}_{\mathcal{H}_{c}}\left(p_{o}\right)-\ddot{s}_{\mathcal{H}_{c}}(p) \underline{\dot{\hat{h}}}(p)\right\|}{\dot{s}_{\mathcal{H}_{c}}^{3}\left(p_{o}\right)} \\
& \underline{u}_{2}(p)=\frac{\ddot{\ddot{h}}(p) \dot{s}_{\mathcal{H}_{c}}\left(p_{o}\right)-\ddot{s}_{\mathcal{H}_{c}}(p) \underline{\dot{\hat{h}}}(p)}{\left\|\underline{\hat{h}}(p) \partial p \dot{s}_{\mathcal{H}_{c}}\left(p_{o}\right)-\ddot{s}_{\mathcal{H}_{c}}(p) \underline{\underline{\dot{h}}}(p)\right\|} .
\end{aligned}
$$

Based on the expression of the rate of change of the arc length of the extended array manifold curves, (28), and the estimation of the first curvature given in (38) we are now in position to 
compare the bearing curves of the various extended array manifolds presented in Section II-A. Note that the analysis in the following section will be limited in the case of linear arrays or equivalent linear arrays (ELA) of planar arrays (see [3]) for the sake of simplicity.

\section{B. Hyperhelical Extended Manifold Curves}

As it has been already said, in the most general case of the extended array manifold curves, the matrix of the linear mapping $\mathrm{A}(p)$ depends on some fixed channel parameters and on the bearing variable $p$. However, if $\mathbb{A}$ is independent of the bearing parameter $p$, then the resulting linear mapping has some extra structure. This structure enables for the analytical evaluation of the entire spectrum of the geometric properties of the extended manifold curve in terms of the corresponding properties of the array manifold curve and the matrix $A$. The detailed analysis of the curvatures of extended manifold curves, resulting from conformal $^{2}$ linear mappings of the array manifold curves will be presented in Appendix A. The most significant result, summarized in Theorem 1, will be given here and used to calculate the curvatures of various hyperhelical extended array manifold curves.

Theorem 1: Let $\mathcal{A} \triangleq\left\{\underline{\mathrm{a}}(p) \in \mathcal{C}^{N}, \forall p: p \in\left[0^{\circ}, 180^{\circ}\right)\right\}$ be the array manifold curve of a linear array of $N$ elements, the shape of which is described by the Cartan Matrix $\mathbb{C}$, containing the array manifold curvatures. Let another manifold curve $\mathcal{H}_{c} \triangleq$ $\left\{\underline{h}(p) \in \mathcal{C}^{Q}, \forall p \in \Omega_{p}\right\}$, related to $\mathcal{A}$ via the linear mapping $\mathcal{T}$ : $\mathcal{C}^{N} \succ \mathcal{C}^{Q}$. That is, $\mathrm{A}(p) \in \mathcal{C}^{Q \times N}$ is the matrix representing the mapping $\mathcal{T}$, such that

$$
\underline{h}(p)=\mathbb{A}(p) \underline{\mathrm{a}}(p)
$$

If $\frac{\partial \mathrm{A}(p)}{\partial p}=\mathbb{O}$ then $\mathbb{A}^{H} \mathbb{A}=\sigma_{\AA}^{2} \rrbracket_{N}$ and the Cartan matrix $\mathbb{C}_{\mathcal{H}_{c}}(p)$ describing the shape of $\mathcal{H}_{c}$ can be estimated as

$$
\mathbb{C}_{\mathcal{H}_{c}}=\frac{1}{\sigma_{\mathrm{A}}} \mathbb{C}
$$

Furthermore, the coordinate vectors $\underline{u}_{i, \mathcal{H}_{c}}(p), i=1 \ldots, d$ of $\mathcal{H}_{c}$ can be expressed as functions of the coordinate vectors $\underline{u}_{i, \mathcal{A}}(p), i=1 \ldots, d$ of $\mathcal{A}$ as follows

$$
\underline{u}_{i, \mathcal{H}_{c}}(p)=\frac{1}{\sigma_{\mathrm{A}}} \mathrm{A} \underline{u}_{i, \mathcal{A}}(p)
$$

The proof of this Theorem and a derivation of closed form formulae for the expression of the coordinate vectors $\underline{u}_{i, \mathcal{H}_{c}}, i=$ $1, \ldots, d$ and the curvatures $\kappa_{i, \mathcal{H}_{c}}, i=1, \ldots, d$ of the extended array manifold curves can be found in Appendix A.

The importance of this theorem lies in the fact that if the conformality assumption is satisfied, then the curvatures of the extended manifold curves are just a scaled version of the curvatures of the original array manifold curves. However, it is known from [3] that the curvatures of the array manifold curves of linear arrays are constant and independent of the bearing variable $p$, that is these curves are of hyperhelical shape. Thus, the extended manifold curves are also of hyperhelical shape. Therefore, the theoretical tools developed so far for the study of the

\footnotetext{
${ }^{2} \mathrm{~A}$ mapping is conformal when it preserves the angles between vectors.
}

hyperhelical array manifold curves [3] can be readily applied to study the extended manifold curves as well.

Three of the extended array manifolds presented in Section II-A are produced by conformal linear mappings, the STAR manifold, the Doppler-STAR manifold and finally the Multi-Carrier-STAR manifold. Using Theorem 1 and the known curvatures of the corresponding array manifold curve, their curvatures can easily be estimated. The required $A$ and $\sigma_{\text {A }}$ parameters for each of these manifold curves are given next. Please note that a proper subscript will be added to the A matrices for each different extended array manifold for the sake of clarity.

1) STAR manifold curves

$$
\begin{aligned}
\mathrm{Al}_{\mathrm{STAR}} & =\rrbracket_{N} \otimes \mathbb{l}^{l} \underline{c} \\
\sigma_{\text {A }} & =\left\|\mathbb{l}^{l} \underline{c}\right\|= \begin{cases}\sqrt{N_{c}}, & 0 \leq l \leq N_{c} \\
\sqrt{2 N_{c}-l}, & N_{c}<l<2 N_{c}\end{cases}
\end{aligned}
$$

2) Doppler-STAR Manifold curves

$$
\begin{aligned}
\mathrm{Al}_{\mathrm{DOP}-\mathrm{STAR}} & =\mathbb{\rrbracket}_{N} \otimes \mathbb{l}^{l} \underline{\underline{c}} \odot \underline{\mathcal{F}}_{D}[n] \\
\sigma_{\text {A }} & = \begin{cases}\sqrt{N_{c}}, & 0 \leq l \leq N_{c} \\
\sqrt{2 N_{c}-l}, & N_{c}<l<2 N_{c}\end{cases}
\end{aligned}
$$

\section{3) Multi-carrier STAR Manifold Curves}

$$
\begin{aligned}
\mathrm{A}_{\mathrm{MC}-\mathrm{STAR}} & =\mathbb{\rrbracket}_{N} \otimes\left(\mathbb{J}^{l} \underline{a}\left[l, F_{k}\right]\right) \\
\sigma_{\mathrm{A}} & = \begin{cases}\sqrt{N_{c} N_{s c}}, & 0 \leq l \leq N_{c} N_{c s} \\
\sqrt{2 N_{c} N_{c s}-l}, & N_{c} N_{c s}<l<2 N_{c} N_{c s}\end{cases}
\end{aligned}
$$

where the various parameters have been defined in Section II-A and the relevant references.

From (42) and (43), it is clear that the parameter $\sigma_{A}$ is the same for the STAR and the Doppler-STAR manifold curves. Consequently, the curvatures and, therefore, the shape of these manifold curves is identical. However, the orientations of the extended manifold curves within the $2 N N_{c}$-dimensional complex space are not identical and this clearly distinguishes the two manifold curves.

The curvatures for both the array and the STAR, DopplerSTAR manifold curves for a symmetrical and a nonsymmetrical linear array have been estimated and their values are plotted in Fig. 2(a) and (b). ${ }^{3}$ Note that the tag "extended curve" in the graphs refers both to the STAR and the Doppler-STAR curves. The number of nonzero curvatures is the same for both the array and the extended array hyperhelical manifold curves which arise from the same array geometry. This implies that both curves lie in a subspace of the overall observation space of the same dimensionality.

It is important to note that if the assumption that "the pathdelay is smaller than the period of a data symbol $T_{c s}$ " is invalid, or equivalently the discretized delay $l$ is greater than the PN-code period $N_{c}$, then only some of the energy of the current data symbol is contained within the tapped delay line (TDL); see Section II-A and [21]. The total energy of a symbol in the TDL is directly connected to the radius of the hypersphere on

${ }^{3}$ Fig. 2(a) has been presented in [30] However this paper was dealing with the STAR manifold exclusively, whereas here it serves as an example for the applicability of our approach. 


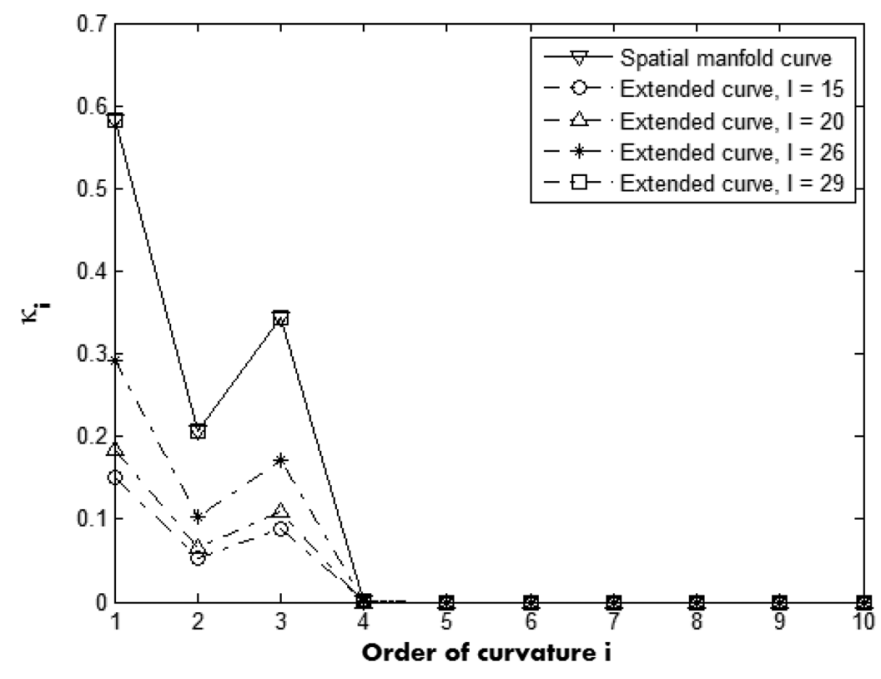

(a)

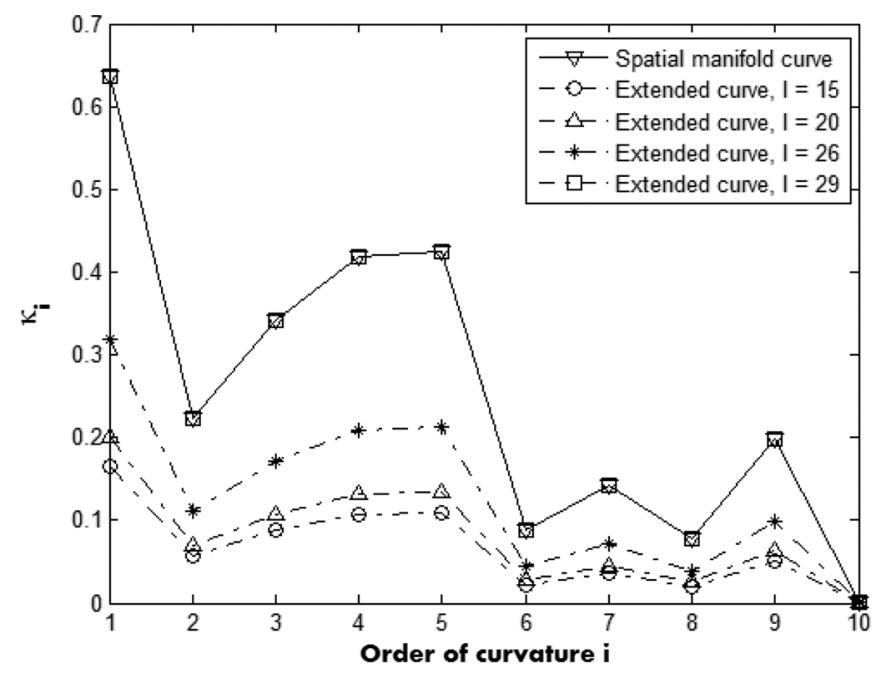

(b)

Fig. 2. Curvatures of conventional and extended hyperhelical manifold curves for a PN-code of period $N_{c}=15$. (a) Symmetrical array at $(-2,-1,0,1,2)$; (b) asymmetrical array at $(-3,-1,0.5,0.9,2.6)$.

which the manifold curve lies. This is the reason why the curvatures of the extended manifold curves increase in magnitude as $l>N_{c}$.

\section{Nonhyperhelical Extended Manifold Curves}

In this subsection, the results of Section III-A will be used to analyze the extended manifold curves of linear arrays of the following:

1) the POLAR-STAR manifold curves,

2) manifold curves of directional elements, which do not preserve the hyperhelical shape of the array manifold curves.

1) POLAR-STAR Manifold Curves: To study the POLARSTAR manifold curves, the overall mapping will be partitioned into two mappings. The first part will provide an intermediate curve (POLAR curve) which will be studied using the proposed framework in Section III-A. The second mapping, from POLAR to POLAR-STAR, will be shown to satisfy the assumptions of Theorem 1 and, thus, this theorem will be applied.

The POLAR manifold vector in the case of crossed-dipole arrays and for coplanar sources can be written as

$$
\underline{h}^{\text {POLAR }}=\left[\mathbb{\natural}_{N} \otimes \underline{\mathrm{q}}(p)\right] \underline{\mathrm{a}}(p)
$$

where $\mathrm{q}(p)$ has been defined in [26]. Based on (45) one may calculate for this intermediate curve

$$
\begin{aligned}
\sigma_{\text {A }} & =\sqrt{V_{x}^{2} \sin ^{2} p \cos ^{2} \gamma+V_{z}^{2} \sin ^{2} \gamma}, \\
\sigma_{\dot{A}} & =V_{x} \cos p \cos \gamma \\
A_{\text {INTER }} & =\mathbb{a}_{N} \otimes \underline{\mathrm{q}}(p)
\end{aligned}
$$

with $V_{x}, V_{z}$ being the sensitivity of the sensors in fields polarized solely in the $x$ and $z$ directions and $\gamma$ being one of the parameters defining the signal polarization (see also [26]).
Furthermore, the POLAR-STAR manifold vector can be expressed as a function of (45) as follows:

$$
\underline{h}^{\text {POLAR-STAR }}=\underbrace{\left[\mathbb{1}_{2 N} \otimes \mathbb{J}^{l} \underline{c}\right]}_{\triangleq A_{\text {POLAR-STAR }}} \underline{h}^{\text {POLAR }} .
$$

Since $A_{\text {POLAR-STAR }}$ is independent of the bearing parameter $p$, Theorem 1 can be applied for the estimation of the geometric parameters of the POLAR-STAR curves based on those of the POLAR manifold curves.

2) Manifold Curves of Arrays of Directional Elements: Consider a linear or ELA having directional elements (i.e., nonisotropic). In this case, the array response vector (weighted array manifold vector) can be expressed as a function of the array manifold vector $\underline{a}(p)$ for isotropic elements as follows:

$$
\underline{\mathrm{a}}_{\mathrm{w}}(p)=\underline{g}(p) \odot \underline{\mathrm{a}}(p)=\mathrm{A}_{\mathrm{w}} \underline{\mathrm{a}}(p), \quad A_{\mathrm{w}} \triangleq \operatorname{diag}\{\underline{g}(p)\}
$$

and $g(p) \in \mathcal{C}^{N}$ is a vector containing the complex-valued gains of each array element. Of course, (48) defines a linear mapping of the array manifold vector.

In Fig. 3(a) and (b), four different manifold curves of a linear array have been studied. The first one is the array manifold curve of a uniform linear array (ULA), the elements of which are positioned at $\underline{r}_{x}=[-2,-1,0,1,2]$ in units of half wavelengths. The second curve is the curve of the same array, with the exception that the elements are now considered to be sensitive to the polarization of the incoming signal, with polarization parameters equal to (see also (17))

$$
V_{x}=0.5, V_{z}=0.5, \gamma=0.1, \eta=0.5
$$

The other two curves are the manifold curves of the same array, when the elements of the array are assumed to have the following gain patterns $g_{1}(p)=\sin p, g_{2}(p)=\frac{\sin \left(p-\frac{\pi}{2}\right)}{\left(p-\frac{\pi}{2}\right)}$.

The dependence of the principal curvature on the directional parameter $p$ implies that specific operational characteristics of 


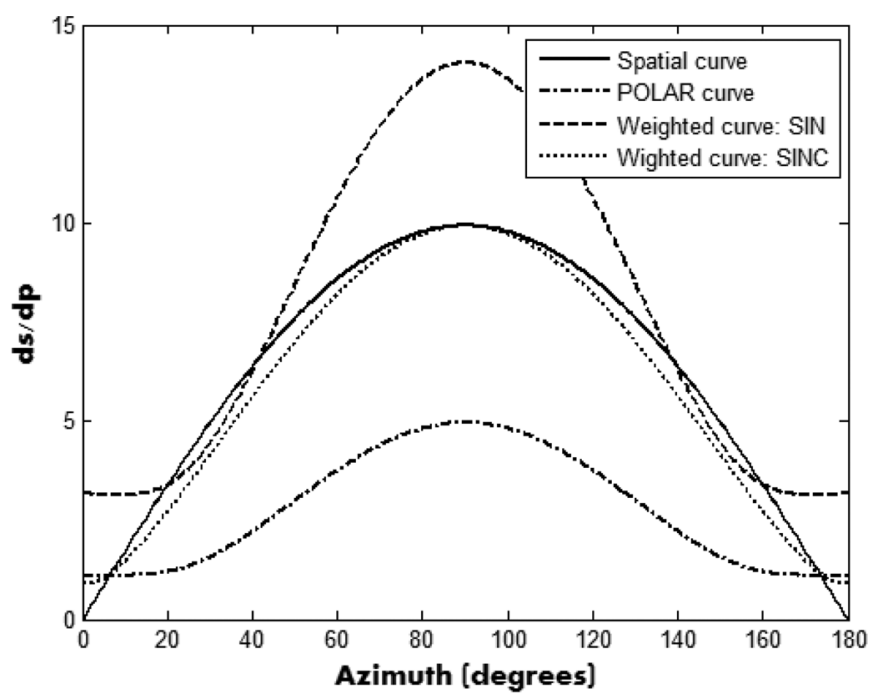

(a)

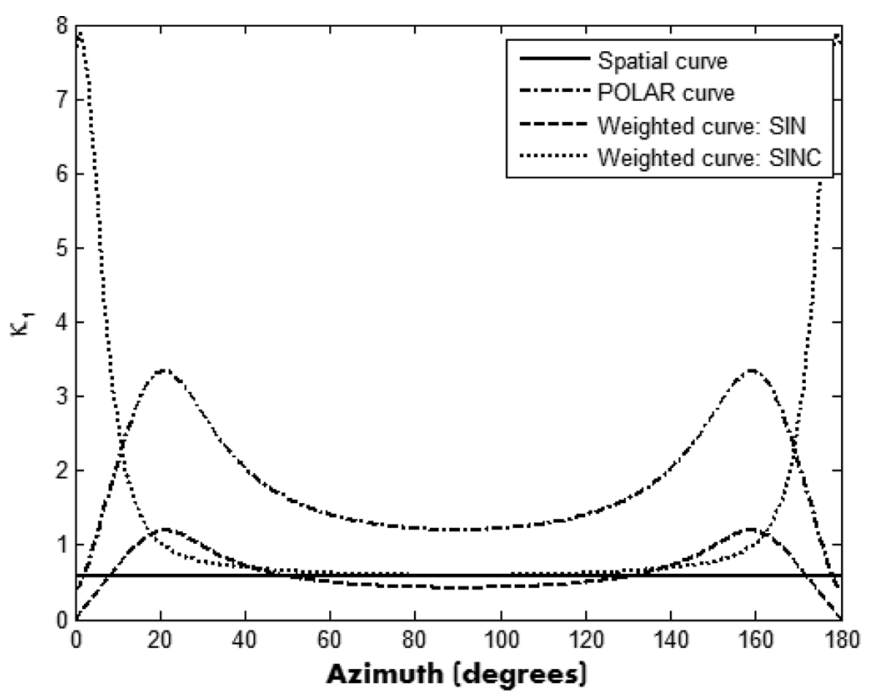

(b)

Fig. 3. Geometric parameters of extended manifold curves. (a) Rate of change of the arc length $\dot{s}(\theta)$; (b) principal curvature $\kappa_{1}$.

the array systems modelled by these manifolds are more sensitive of the direction of arrival of the signals. For example, the theoretical resolution threshold [3], [10] and the Cramér-Rao lower bound [3], [31] are dependent on the rate of change of the arc length, which is $p$-dependent even in the hyperhelical manifold curves, but also on the principal curvature.

\section{EXTENDED ARRAY MANIFOLD SURFACES}

In the previous section, the geometric properties of the extended array manifold curves have been studied as a function of the geometric properties of the array manifold curves and the nature of the extension. The focus is now redirected to 2parameter extended array manifolds, i.e., extended array manifold surfaces.

Assuming that the extended array manifold vector is now dependent on both bearing parameters $p$ and $q$, the extended array manifold is defined as

$$
\mathcal{M}_{\mathcal{H}} \triangleq\left\{\underline{h}(p, q), \forall(p, q) \in \Omega_{p, q}\right\}
$$

where, according to (20), $\underline{h}(p, q)=\mathrm{A}(p, q) \underline{\mathrm{a}}(p, q)$.

The objective of this section is the investigation of the most important geometric properties of the extended array manifold surfaces, which were presented in Section I. In detail, a relationship will be established between the properties of the extended array manifold surfaces and the corresponding properties of the array manifold surfaces. This relationship, will not only allow for an efficient computation of the geometric properties of the extended manifold surfaces, given the existing results for the array manifold surfaces, but will also provide an insight into the geometrical differences and similarities between the two manifolds.

Note that, in the analysis that follows, every variable with a subscript $\mathcal{H}$ will refer to the geometric properties of the extended array manifold surface $\mathcal{M}_{\mathcal{H}}$, whereas variables without a subscript will refer to the properties of the array manifold surface $\mathcal{M}$, where

$$
\mathcal{M} \triangleq\left\{\underline{\mathrm{a}}(p, q) \in \mathcal{C}^{N}, \forall(p, q):(p, q) \in \Omega\right\} \text {. }
$$

\section{A. "Conformal" Extended Array Manifold Surfaces}

Consider an array of $N$ omni-directional elements. Its response is described by the vector $\underline{\mathrm{a}}(p, q)$ and the associated array manifold is a surface embedded in an $N$-dimensional complex space. Moreover, consider a linear mapping $\mathcal{T}: \mathcal{C}^{N} \mapsto \mathcal{C}^{Q}$ (i.e., from an $N$-dimensional space to a $Q$-dimensional space, $Q \geq N$ ), described by a matrix A, with

$$
\mathrm{A}=\mathbb{\rrbracket}_{N} \otimes \underline{z}(p, q)
$$

according to Section II-B. In this section, the case where

$$
\frac{\partial \mathrm{A}}{\partial p}=\frac{\partial \mathrm{A}}{\partial q}=\mathbb{O}
$$

will be investigated. This condition will be relaxed in the following section, when more general mappings will be considered. For the rest of this paper, any extended array manifold surface satisfying (53) will be called conformal extended array manifold surface, as it is produced by conformal complex mappings acting on the array manifold surfaces.

The tangent plane at a point $\underline{h}(p, q)$ on the manifold surface is a plane surface spanned by the two tangent vectors $\underline{\dot{h}}_{p}$ and $\underline{\underline{h}}_{q}$. Therefore, if $\mathbb{T}_{\mathcal{H}} \triangleq\left[\underline{\underline{h}}_{p}, \underline{\underline{h}}_{q}\right]$, is a basis for the tangent plane of the extended array manifold surface and $\mathbb{T} \triangleq\left[\underline{a}_{p}, \underline{\dot{a}}_{q}\right]$ is a basis for the array manifold surface, then these two bases are related as follows:

$$
\mathbb{T}_{\mathcal{H}}=\left[\underline{\dot{h}}_{p}, \underline{\dot{h}}_{q}\right]=\left[\mathrm{A} \underline{\dot{a}}_{p}, \text { A } \underline{\dot{a}}_{q}\right]=\mathrm{AT} .
$$

From (54), one can deduce that the tangent plane of the extended array manifold surface at a specific point $\underline{h}(p, q)$ will be rotated with regards to the tangent plane of the array manifold surface at 
the corresponding point $\underline{\mathrm{a}}(p, q)$. This result was expected since the effect of the linear mapping $\mathcal{T}$ results in a rotation of the whole array manifold surface. Note, however, that this is not a trivial rotation such as, for example the rotation of a circle in a 3-dimensional real space, but it can be viewed as the rotation of a circle formerly lying on a 2-dimensional plane, into the full 3-dimensional space.

Furthermore, based on (54) the relationship connecting the two manifold metrics $\mathbb{G}$ and $\mathbb{G}_{\mathcal{H}}$ can be derived:

$$
\begin{aligned}
& \mathbb{G}_{\mathcal{H}}=\operatorname{Re}\left\{\left(\mathbb{T}_{\mathcal{H}}\right)^{H} \mathbb{T}_{\mathcal{H}}\right\}=\operatorname{Re}\left\{(\mathrm{A} \mathbb{T})^{H} \mathrm{~A} \mathbb{T}\right\} \\
& =\operatorname{Re}\left\{\mathbb{T}^{H}\left(\sigma_{\AA}^{2} \rrbracket_{N}\right) \mathbb{T}\right\}=\sigma_{\AA}^{2} \operatorname{Re}\left\{\mathbb{T}^{H} \mathbb{T}\right\}=\sigma_{\AA}^{2} \mathbb{G} .
\end{aligned}
$$

The elements of the manifold metric are used to measure trajectories on the manifold surface via the concept of the first fundamental form $\mathcal{I}$

$$
\mathcal{I} \triangleq d \underline{\mathrm{p}}^{T} \mathbb{G} d \underline{\mathrm{p}}
$$

where $\mathrm{p}(s)=[p(s), q(s)]^{T}$. Thus, (55) and (56) imply that for the same infinitesimal increment $d p, d q$ of the directional parameters, the distance between $\underline{h}(p, q)$ and $\underline{h}(p+d p, q+d q)$ will be $\sigma_{\mathcal{A}}$ times the distance between the corresponding array manifold vectors. Moreover, the area with sides $d p, d q$ is mapped via the extended array manifold vector $\underline{h}(p, q)$ onto an infinitesimal surface of an area $\sigma_{\AA}^{2}$ larger than the area of the surface which the array manifold vector produces. This is consistent with the result of the previous section that the new manifold is on a sphere with a radius $\sigma_{\text {A }}$ times larger than that of the associated array manifold.

The Christoffel Matrices of the first kind $\boldsymbol{\Gamma}_{1 p, \mathcal{H}}$ and $\boldsymbol{\Gamma}_{1 q, \mathcal{H}}$ of the extended manifold surfaces are related to the corresponding matrices $\boldsymbol{\Gamma}_{1 p}$ and $\boldsymbol{\Gamma}_{1 q}$ of the array manifold surfaces as follows:

$$
\begin{aligned}
\Gamma_{1 \zeta, \mathcal{H}} & =\operatorname{Re}\left\{\mathbb{T}_{\mathcal{H}}^{H} \dot{\mathbb{T}}_{\zeta, \mathcal{H}}\right\}=\operatorname{Re}\left\{(\mathrm{AT})^{H} \AA \dot{\mathbb{T}}_{\zeta}\right\} \\
& =\sigma_{\AA}^{2} \operatorname{Re}\left\{\mathbb{T}^{H} \dot{\mathbb{T}}_{\zeta}\right\}=\sigma_{\AA}^{2} \boldsymbol{\Gamma}_{1 \zeta}
\end{aligned}
$$

where

$$
\dot{\mathbb{T}}_{\zeta} \triangleq \frac{\partial \mathbb{T}}{\partial \zeta}, \quad \dot{\mathbb{T}}_{\zeta, \mathcal{H}} \triangleq \frac{\partial \mathbb{T}_{\mathcal{H}}}{\partial \zeta}
$$

with $\zeta=p, q$, while the Christoffel matrices of the second kind are identical.

$$
\Gamma_{2 \zeta, \mathcal{H}}=\left(\mathbb{G}_{\mathcal{H}}\right)^{-1} \boldsymbol{\Gamma}_{1 \zeta, \mathcal{H}}=\frac{1}{\sigma_{\AA}^{2}} \mathbb{G}^{-1} \sigma_{\AA}^{2} \boldsymbol{\Gamma}_{1 \zeta}=\boldsymbol{\Gamma}_{2 \zeta} .
$$

The Christoffel matrices are used to calculate the infinitesimal variance of the tangent plane as the point $\underline{h}(p, q)$ moves on the manifold surface by $d \underline{h}=\underline{\hat{h}}_{p} d p+\underline{\hat{h}}_{q} d q$, that is

$d \mathbb{T}_{\mathcal{H}}=\mathbb{T}_{\mathcal{H}}\left(\boldsymbol{\Gamma}_{2 p, \mathcal{H}} d p+\boldsymbol{\Gamma}_{2 q, \mathcal{H}} d q\right) \mathrm{A} \mathbb{\mathbb { T }}\left(\boldsymbol{\Gamma}_{2 p} d p+\boldsymbol{\Gamma}_{2 q} d q\right)=\mathrm{A} d \mathbb{\mathbb { T }}$.

However, since the shape of the actual surface has not changed, the only difference $d \mathbb{T}_{\mathcal{H}}$ in the change of the vectors spanning the tangent plane in the case of the extended array manifold surface (compared to $d \mathbb{T}$ of the array manifold surface) is due to the original transformation of the tangent plane, which is described in (54).

The final geometric property of surfaces, which will be examined is that of the Gaussian curvature. The sign of the Gaussian curvature of the array manifold surface at a point $\underline{h}(p, q)$ provides an indication of the local shape of the surface at the neighbourhood of this point. Since the shape of the array manifold surface has already been studied in the literature, it would be convenient to be able to link the shape of the extended array manifold surfaces to the corresponding conventional ones. The following theorem, Theorem 2, provides this connection.

Theorem 2: Let $\mathcal{M} \triangleq\left\{\underline{\mathrm{a}}(p, q) \in \mathcal{C}^{N}, \forall(p, q) \in \Omega_{p, q}\right\}$ be the array manifold surface of an array of $N$ elements, the shape of which is described, locally at a point $\underline{\mathrm{a}}(p, q)$, by the Gaussian curvature $K_{g}(p, q)$. Let $\mathcal{M}_{\mathcal{H}} \triangleq\left\{\underline{h}(p, q) \in \mathcal{C}^{Q}, \forall(p, q) \in \Omega_{p, q}\right\}$ be an extended array manifold surface, related to $\mathcal{M}$ via the linear mapping $\mathcal{T}: \mathcal{C}^{N} \rightarrow \mathcal{C}^{Q}$. That is, $A \in \mathcal{C}^{Q \times N}$ is the matrix representing the mapping $\mathcal{T}, \underline{h}(p)=\mathbb{A}(p, q) \underline{\mathrm{a}}(p, q)$.

If $\frac{\partial \mathcal{A}(p, q)}{\partial p}=\frac{\partial \mathcal{A}(p, q)}{\partial q}=\mathbb{O}$ and $\AA^{H} \AA$

1) the Gaussian curvature $K_{g, \mathcal{H}}$ of the extended manifold surface $\mathcal{M}_{\mathcal{H}}$ can be computed as

$$
K_{g, \mathcal{H}}(p, q)=\frac{1}{\sigma_{\text {A }}^{2}} K_{g}(p, q) ;
$$

2) The shape of $\mathcal{M}_{\mathcal{H}}$ at the neighborhood of a point $\underline{h}(p, q)$ has locally the same shape, as the array manifold surface $\mathcal{M}$ has at a neighborhood around $\underline{\mathrm{a}}(p, q)$.

Proof: The local shape of a surface at the neighbourhood of a point $\underline{h}\left(p_{o}, q_{o}\right)$ can be determined by the sign of the Gaussian Curvature at that point. Therefore, it is sufficient to prove (61). For the rest of this proof, the dependence of all magnitudes on the parameters $p$ and $q$ will be dropped for notational convenience.

An expression for the Gaussian curvature of the surface $\mathcal{M}_{\mathcal{H}}$, based on the intrinsic geometry of the surface, is [32]

$$
\begin{aligned}
& K_{g, \mathcal{H}}(p, q)=-\frac{1}{\sqrt{\operatorname{det}\left\{\mathbb{G}_{\mathcal{H}}\right\}}} \\
& \quad\left(\frac{d\left(\frac{\sqrt{\operatorname{det}\left\{\mathbb{G}_{\mathcal{H}}\right\}}}{g_{p p, \mathcal{H}}} \Gamma_{p q, \mathcal{H}}^{q}\right)}{d p}-\frac{d\left(\frac{\sqrt{\operatorname{det}\left\{\mathbb{G}_{\mathcal{H}}\right\}}}{g_{p p, \mathcal{H}}} \Gamma_{p p, \mathcal{H}}^{q}\right)}{d q}\right)
\end{aligned}
$$

where $g_{p p, \mathcal{H}} \triangleq \underline{\dot{h}}_{p}^{H} \underline{h}_{p}=\sigma_{\text {ค }}^{2} \underline{\dot{\mathrm{a}}}_{p}^{H} \underline{\dot{\mathrm{a}}}_{p}=\sigma_{\text {А }}^{2} g_{p p}$ and

$$
\Gamma_{2 \zeta, \mathcal{H}}=\left[\begin{array}{ll}
\Gamma_{p p, \mathcal{H}}^{\zeta} & \Gamma_{p p, \mathcal{H}}^{\zeta} \\
\Gamma_{q p, \mathcal{H}}^{\zeta} & \Gamma_{q q, \mathcal{H}}^{\zeta}
\end{array}\right]=\left[\begin{array}{cc}
\Gamma_{p p}^{\zeta} & \Gamma_{p p}^{\zeta} \\
\Gamma_{q p}^{\zeta} & \Gamma_{q q}^{\zeta}
\end{array}\right], \quad \zeta=p, q .
$$

Thus, by substituting into (62)

$$
\begin{aligned}
K_{g, \mathcal{H}}= & -\frac{1}{\sqrt{\sigma_{\mathrm{A}}^{4} \operatorname{det}\{\mathbb{G}\}}} \\
& \cdot\left(\frac{d\left(\frac{\sigma_{\mathrm{A}}^{2} \sqrt{\operatorname{det}\{\mathbb{G}\}}}{\sigma_{\mathrm{A}}^{2} g_{p p}} \Gamma_{p q}^{q}\right)}{d p}-\frac{d\left(\frac{\sigma_{\mathrm{A}}^{2} \sqrt{\operatorname{det}\{\mathbb{G}\}}}{\sigma_{\mathrm{A}}^{2} g_{p p}} \Gamma_{p p}^{q}\right)}{d q}\right)
\end{aligned}
$$




$$
\begin{aligned}
= & -\frac{1}{\sigma_{\AA}^{2}} \frac{1}{\sqrt{\operatorname{det}\{\mathbb{G}\}}} \\
& \cdot\left(\frac{d\left(\frac{\sqrt{\operatorname{det}\{\mathbb{G}\}}}{g_{p p}} \Gamma_{p q}^{q}\right)}{d p}-\frac{d\left(\frac{\sqrt{\operatorname{det}\{\mathbb{G}\}}}{g_{p p}} \Gamma_{p p}^{q}\right)}{d q}\right) \\
= & \frac{1}{\sigma_{\AA}^{2}} K_{g} .
\end{aligned}
$$

This relationship between the two Gaussian curvatures reflects the fact that the extended array manifold surface $\mathcal{M}_{\mathcal{H}}$ lies on a hypersphere of radius $\sigma_{\text {A }}$ times larger than that on which the array manifold surface $\mathcal{M}$ lies.

Finally, let us assume that there exists an array manifold curve $\mathcal{A}$ on the array manifold surface $\mathcal{M}$ and the corresponding, via a linear mapping $\mathcal{T}$ which satisfies the conditions of Section II, extended array manifold curve $\mathcal{A}_{\mathcal{H}}$ on $\mathcal{M}_{\mathcal{H}}$. The properties of the extended manifold curves have been extensively studied in Section III. However, for curves lying on surfaces, another geometric property of interest is the geodesic curvature $\kappa_{g}$ of the curve. If the geodesic curvature of a curve $\mathcal{A}$ on a surface $\mathcal{M}$ is equal to zero throughout its length, then this curve defines the path of minimum length on $\mathcal{M}$, which connects the two end points of $\mathcal{A}$. It is interesting to examine how the geodesic curvature $\kappa_{g, \mathcal{H}}(s)$ of the extended manifold curve $\mathcal{A}_{\mathcal{H}}$ is connected to the geodesic curvature $\kappa_{g}(s)$ of the manifold curve $\mathcal{A}$.

Let us consider the formula for the geodesic curvature given in [3].

$$
\begin{aligned}
\kappa_{g}(s)=\sqrt{\operatorname{det}\{\mathbb{G}(s)\}} \frac{\partial}{\partial s}^{T}\left[\begin{array}{cc}
0 & 1 \\
-1 & 0
\end{array}\right] \\
\cdot\left(\frac{\partial^{2} \underline{\mathrm{p}}}{\partial s^{2}}+\left(\Gamma_{2 p} \frac{\partial p}{\partial s}+\Gamma_{2 q} \frac{\partial q}{\partial s}\right) \frac{\partial \underline{\mathrm{p}}}{\partial s}\right) .
\end{aligned}
$$

For an extended array manifold curve, (64) can be reformulated as

$$
\begin{aligned}
& \kappa_{g, \mathcal{H}}(s)=\sqrt{\operatorname{det}\left\{\mathbb{G}_{\mathcal{H}}(s)\right\}} \frac{\partial \underline{\mathrm{p}}_{\mathcal{H}}{ }^{T}}{\partial s}\left[\begin{array}{cc}
0 & 1 \\
-1 & 0
\end{array}\right] \\
& \times\left(\frac{\partial^{2} \underline{\mathrm{p}}_{\mathcal{H}}}{\partial s^{2}}+\left(\boldsymbol{\Gamma}_{2 p, \mathcal{H}} \frac{\partial p_{\mathcal{H}}}{\partial s}+\boldsymbol{\Gamma}_{2 q, \mathcal{H}} \frac{\partial q_{\mathcal{H}}}{\partial s}\right) \frac{\partial \underline{\mathrm{p}}_{\mathcal{H}}}{\partial s}\right) .
\end{aligned}
$$

Since the objective is to compare two manifold curves which have, in general, different lengths but the same domain for the bearing parameters $p$ and $q$, it is more convenient to consider (64) and (65) as functions of $p$ and $q$ and not $s$. In other words, the point $\underline{\mathrm{a}}\left(p_{o}, q_{o}\right)$ on $\mathcal{A}$ (and of course on $\left.\mathcal{M}\right)$ is mapped via $\mathcal{T}$ onto the point $\underline{h}\left(p_{o}, q_{o}\right)$ on $\mathcal{A}_{\mathcal{H}}$ (and $\left.\mathcal{M}_{\mathcal{H}}\right)$. However, $\underline{\mathrm{a}}\left(s_{o}\right)$ is not mapped onto $\underline{h}\left(s_{O}\right)$, since as it was shown in Section III the total manifold curve length of $\mathcal{A}$ is different than that of $\mathcal{A}_{\mathcal{H}}$.

For the extended array manifold curve of (65)

$$
\frac{\partial \underline{\mathrm{p}}_{\mathcal{H}}}{\partial s}=\left(\frac{\partial s}{\partial \underline{\mathrm{p}}_{\mathcal{H}}}\right)^{-1}=\left[\frac{1}{\left\|\underline{\dot{\hat{h}}}_{p}\right\|}, \frac{1}{\left\|\underline{\dot{h}}_{q}\right\|}\right]^{T}=\frac{1}{\sigma_{\text {A }}} \frac{\partial \underline{\mathrm{p}}}{\partial s}
$$

and

$$
\frac{\partial^{2} \underline{\mathrm{p}}_{\mathcal{H}}}{\partial s^{2}}=\frac{\partial}{\partial s}\left(\frac{\partial \underline{\mathrm{p}}_{\mathcal{H}}}{\partial s}\right)=\frac{1}{\sigma_{\mathcal{A}}} \frac{\partial^{2} \underline{\mathrm{p}}}{\partial s^{2}} .
$$

Therefore

$$
\begin{aligned}
& \kappa_{g, \mathcal{H}}(p, q)=\sigma_{\AA}^{2} \sqrt{\operatorname{det}\{\mathbb{G}(s)\}} \frac{1}{\sigma_{\text {A }}} \frac{\partial \underline{\mathrm{p}}^{T}}{\partial s}\left[\begin{array}{cc}
0 & 1 \\
-1 & 0
\end{array}\right] \\
& \frac{1}{\sigma_{\text {A }}}\left(\frac{\partial^{2} \underline{\mathrm{p}}}{\partial s^{2}}+\left(\boldsymbol{\Gamma}_{2 p} \frac{\partial p_{\mathcal{H}}}{\partial s}+\boldsymbol{\Gamma}_{2 q} \frac{\partial q}{\partial s}\right) \frac{\partial \underline{\mathrm{p}}}{\partial s}\right)=\kappa_{g}(p, q) .
\end{aligned}
$$

Note, of course that since the geodesic curvature is a property associated with the manifold curve, it depends on one parameter only which is consistent with the notational convention $\kappa_{g}(p, q)$ since there is a dependency between $p$ and $q$. The main conclusion drawn from (68) is that geodesic array manifold curves are mapped to geodesic extended manifold curves.

\section{B. "Nonconformal" Extended Manifold Surfaces}

In the previous section, the differential geometric properties of the extended array manifold surfaces arising from conformal complex mappings (i.e., A independent of $p, q$ ) of the array manifold surfaces have been examined. The attention is now turned to extended array manifold surfaces which are the product of nonconformal mappings. Namely, conditions (53) are relaxed and now the matrix $\mathbb{A}=A(p, q)$ is a function of both $p$ and $q$. These extended array manifold surfaces will be referred to for the rest of this paper as nonconformal extended array manifold surfaces. It is expected that the nonconformality of the complex mapping will deform the array manifold surface and the relatively simple connection between the geometric properties of the two surfaces will not be present any more.

The investigation will commence with the tangent plane at some point $\underline{h}(p, q)$ of the extended manifold surface. Similarly to (54), which is true for conformal mappings, $\mathbb{T}_{\mathcal{H}}$ for nonconformal mappings can be expressed as follows:

$$
\begin{aligned}
& \mathbb{T}_{\mathcal{H}}=\left[\underline{\dot{h}}_{p}, \dot{h}_{q}\right]=\left[\dot{\mathrm{A}}_{p} \underline{\mathrm{a}}+\mathrm{A} \underline{\dot{a}}_{p}, \dot{\mathrm{A}}_{q} \underline{\mathrm{a}}+\mathrm{A} \underline{\dot{a}}_{q}\right] \\
& =\mathrm{AT}+\dot{\mathrm{A}}_{p q}\left(\mathbb{D}_{2} \otimes \underline{\mathrm{a}}\right)
\end{aligned}
$$

where

$$
\dot{\mathrm{A}}_{p q} \triangleq\left[\dot{\mathrm{A}}_{p}, \dot{\mathrm{A}}_{q}\right]=\left[\frac{\partial \mathrm{A}}{\partial p}, \frac{\partial \mathrm{A}}{\partial q}\right] .
$$

Note that now the basis of the tangent plane of the extended manifold surface is comprised of two terms. The first term is identical to (54), while the second one represents the deformation of the surface because of the dependence of the complex mapping on the bearing parameters $p$ and $q$.

Based on (69) the relationship connecting the two manifold metrics $\mathbb{G}$ and $\mathbb{G}_{\mathcal{H}}$ can be derived:

$$
\begin{aligned}
& \mathbb{G}_{\mathcal{H}}=\operatorname{Re}\left\{\left(\mathbb{\mathbb { T }}_{\mathcal{H}}\right)^{H} \mathbb{T}_{\mathcal{H}}\right\} \\
& =\operatorname{Re}\left\{\left(A \mathbb{T}+\dot{\mathrm{A}}_{p q} \mathbb{D}_{2} \otimes \underline{\mathrm{a}}\right)^{H}\left(\mathrm{~A} \mathbb{T}+\dot{\mathrm{A}}_{p q} \mathbb{D}_{2} \otimes \underline{\mathrm{a}}\right)\right\} \\
& =\operatorname{Re}\left\{(\boldsymbol{A} \mathbb{T})^{H} \mathrm{~A} \mathbb{T}+\left(\dot{\mathrm{A}}_{p q} \mathbb{\rrbracket}_{2} \otimes \underline{\mathrm{a}}\right)^{H} \dot{\mathrm{A}}_{p q} \rrbracket_{2} \otimes \underline{\mathrm{a}}\right\} \\
& +\operatorname{Re}\left\{2\left(\dot{\mathrm{A}}_{p q} \rrbracket_{2} \otimes \underline{\mathrm{a}}\right)^{H} \mathrm{AT} \mathrm{T}\right\}^{0} \\
& =\sigma_{\AA}^{2} \mathbb{G}+N \mathbb{G}_{\underline{z}}
\end{aligned}
$$


where, in similarity to the definitions of $\mathbb{G}$ and $\mathbb{G}_{\mathcal{H}}, \mathbb{G}_{\underline{z}}$ is defined as

$$
\mathbb{G}_{\underline{z}} \triangleq\left[\begin{array}{cc}
\dot{\underline{z}}_{p}^{H} \underline{\underline{z}}_{p} & \operatorname{Re}\left\{\underline{\dot{z}}_{p}^{H} \underline{\dot{z}}_{q}\right\} \\
\operatorname{Re}\left\{\underline{\dot{z}}_{q}^{H} \dot{\underline{z}}_{p}\right\} & \underline{\dot{z}}_{q}^{H} \underline{\dot{z}}_{q}
\end{array}\right] .
$$

Let us compare (71) with (55). For an nonconformal extended array manifold surface there are two terms contributing to the manifold metric $\mathbb{G}_{\mathcal{H}}$. The first term is the same as the one in (55) and expresses the change of dimensionality of the embedding complex space. The second term $N \mathbb{G}_{\underline{z}}$, however, is related to the dependence of the mapping on the directional parameters and can be visualized as the manifold metric $\mathbb{G}_{\underline{z}}$ of a second surface, whose parametric equation is given by

$$
\mathcal{M}_{\underline{z}}=\left\{\underline{z}(p, q) \in \mathcal{C}^{\frac{Q}{N}},(p, q) \in \Omega_{p, q}\right\} .
$$

Note, however, that the manifold surfaces are embedded in complex spaces of different dimensions, that is $\mathcal{M} \subset \mathcal{C}^{N}, \mathcal{M}_{\mathcal{H}} \subset$ $\mathcal{C}^{Q}$ and $\mathcal{M}_{\underline{z}} \subset \mathcal{C}^{\frac{Q}{N}}$. Therefore, another interpretation of the extended array manifold surface is that the array manifold vector has acted on $\mathcal{M}_{\underline{z}}$ to produce $\mathcal{M}_{\mathcal{H}}$. However, the properties of this new mapping are not entirely dual to those of $\mathcal{T}$ and this can be deduced from the first term on the right-hand side of (69), where the term $\dot{\mathrm{A}}_{p q}=\left[\mathbb{1}_{N} \otimes \underline{\dot{\dot{x}}}_{p}, \rrbracket_{N} \otimes \underline{\dot{\dot{x}}}_{q}\right]$ appears instead of $\left[\underline{\dot{z}}_{p}, \dot{\underline{x}}_{q}\right]$ which would be the dual of $\mathbb{T}$. This, nevertheless, is simply related to the structure of the output of the Tapped Delay Line used in the literature so far.

Let us proceed next to the Christoffel matrices of the first and second kind. Following a detailed analysis which can be found in Appendix B

$$
\begin{aligned}
\Gamma_{1 \zeta, \mathcal{H}}= & \operatorname{Re}\left\{\mathbb{T}_{\mathcal{H}}^{H} \dot{\mathbb{T}}_{\zeta, \mathcal{H}}\right\} \\
= & \operatorname{Re}\left\{\mathbb{T}_{\underline{z}}^{H} \underline{z \dot{\mathrm{a}}_{\zeta}^{H}} \mathbb{\mathbb { T }}+\underline{\dot{z}}_{\zeta}^{H} \underline{z} \widetilde{\mathbb{G}}+\dot{\mathbb{T}}_{\zeta}^{H} \underline{\mathrm{a} z}^{H} \mathbb{T}_{\underline{z}}\right\} \\
& +N \Gamma_{1 \zeta, \underline{z}}+\sigma_{\text {A }}^{2} \boldsymbol{\Gamma}_{1 \zeta}
\end{aligned}
$$

where

$$
\mathbb{T}_{\underline{z}} \triangleq\left[\underline{\dot{z}}_{p}, \dot{\dot{z}}_{q}\right]
$$

is the tangent matrix of $\mathcal{M}_{\underline{z}}$,

$$
\begin{gathered}
\dot{\mathbb{T}}_{\zeta, \underline{z}} \triangleq\left[\underline{\ddot{z}}_{p \zeta}, \ddot{\ddot{z}}_{q \zeta}\right] \\
\Gamma_{1 \zeta, \underline{z}} \triangleq \operatorname{Re}\left\{\dot{\mathbb{T}}_{\zeta, \underline{z}}^{H} \mathbb{T}_{\underline{z}}\right\}
\end{gathered}
$$

is the Christoffel matrix of the first kind for $\mathcal{M}_{\underline{z}}$ and, finally

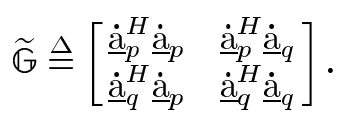

Again, the pattern which appeared initially in the study of the extended manifold curves and next in the tangent matrix $\mathbb{T}_{\mathcal{H}}$ and the manifold metric $\mathbb{G}_{\mathcal{H}}$ is present. On the right hand size of (74) there exist two terms, which are properly scaled versions of the Christoffel matrix of the first kind $\boldsymbol{\Gamma}_{1 \zeta, \mathcal{H}}$ of the extended array manifold surface $\mathcal{M}_{\mathcal{H}}$ and the Christoffel matrix of the first kind $\Gamma_{1 \zeta, \underline{z}}$ of $\mathcal{M}_{\underline{z}}$. The remaining terms

$$
\operatorname{Re}\left\{\mathbb{T}_{\underline{z}}^{H} \underline{z \dot{a}}_{\zeta}^{H} \mathbb{T}+\underline{\dot{z}}_{\zeta}^{H} \underline{z} \widetilde{\mathbb{G}}+\dot{\mathbb{T}}_{\zeta}^{H} \underline{\mathrm{a} z}^{H} \mathbb{T}_{\underline{z}}\right\}
$$

are due to the fact that the reference point of the array system is taken to be the array centroid. This guarantees that
$\underline{1}_{N}^{T}\left[\underline{r}_{x}, \underline{r}_{y}, \underline{r}_{z}\right]=\underline{0}_{3}^{T}$ and therefore $\underline{\dot{a}}_{\zeta}^{H} \underline{\mathrm{a}}=0, \zeta=p, q$ but does not guarantee that the tangent vectors $\underline{\dot{z}}_{\zeta}(p, q), \zeta=p, q$ at every point $\underline{z}(p, q)$ of the manifold surface $\mathcal{M}_{\underline{z}}$ will be normal to the surface.

Finally, the Christoffel matrix of the second kind for the extended manifold surface is related to the respective matrix of the array manifold surface as follows:

$$
\begin{aligned}
& \Gamma_{2 \zeta, \mathcal{H}} \\
& =\left(\mathbb{G}_{\mathcal{H}}\right)^{-1} \boldsymbol{\Gamma}_{1 \zeta, \mathcal{H}} \\
& =\frac{\sigma_{\AA}^{2} \operatorname{det}\{\mathbb{G}\} \mathbb{G}^{-1}+N \operatorname{det}\left\{\mathbb{G}_{\underline{z}}\right\} \mathbb{G}_{\underline{z}}^{-1}}{N^{2} \operatorname{det}\left\{\mathbb{G}_{\underline{z}}\right\}+\sigma_{\mathrm{A}}^{4} \operatorname{det}\{\mathbb{G}\}+N \sigma_{\mathrm{A}}^{2} \operatorname{Tr}\left\{\operatorname{det}\left\{\mathbb{G}_{\underline{z}}\right\} \mathbb{G}_{\mathbb{G}_{\underline{z}}^{-1}}^{-1}\right\}} \\
& \times\left(\operatorname{Re}\left\{\mathbb{T}_{\underline{z}}^{H} \underline{z \dot{\mathrm{a}}}_{\zeta}^{H} \mathbb{T}+\underline{\dot{\dot{x}}}_{\zeta}^{H} \underline{z} \widetilde{\mathbb{G}}+\dot{\mathbb{T}}_{\zeta}^{H} \underline{\mathrm{a} z}^{H} \mathbb{T}_{\underline{z}}\right\}\right. \\
& \left.+N \Gamma_{\underline{z}, 1 \zeta}+\sigma_{A}^{2} \Gamma_{1 \zeta}\right) .
\end{aligned}
$$

\section{CONCLUSION}

In this paper the concept of the extended array manifold has been introduced and its functional dependence on the array manifold has been investigated. In particular, the geometric properties of extended array manifold curves and extended manifold surfaces have been studied as a function of the corresponding properties of the array manifold curves and surfaces. The analytic expressions which were derived for the geometric properties of the extended array manifold can be used to investigate the properties of a wide family of array manifolds used to describe numerous architectures that employ arrays. Thus, the work presented in this paper provides a theoretical framework that has the potential to express, analyze or design more complex array and wireless systems by analyzing or designing curves or surfaces embedded in a multidimensional complex space.

\section{APPENDIX A \\ PROOF OF THEOREM I}

The general formulae for calculating the $i$ th coordinate vector and curvature can be found in [3] and [7].

\section{A. ith Coordinate Vector and Curvature}

Based on the results for the first and second order coordinate vectors and curvatures, the emerging pattern of the formulae leads to the following expressions:

$$
\begin{aligned}
\underline{u}_{i}= & \frac{j^{i}}{\sigma_{\text {A }}^{i} \kappa_{1} \kappa_{2} \ldots \kappa_{i-1}} \text { A } \\
& \cdot\left[\left(\sum_{n=1}^{\left\lfloor\frac{i-1}{2}\right\rfloor+1}(-1)^{n-1} b_{i-1, n} \underline{\underline{r}}^{i-2 n+2}\right) \odot \underline{\mathrm{a}}\right] \\
\kappa_{i}= & \frac{\sigma_{\AA}^{-i}}{\kappa_{1} \kappa_{2} \ldots \kappa_{i-1}}\left\|\sum_{n=1}^{\left\lfloor\frac{i}{2}\right\rfloor+1}(-1)^{n-1} b_{i, n} \underline{\hat{r}}^{i-2 n+3}\right\|
\end{aligned}
$$

where

$$
\left.\begin{array}{c}
b_{i, n}=b_{i-1, n}+\sigma_{\AA}^{2} \kappa_{i-1}^{2} b_{i-2, n-1}, \quad i \geq 1 \\
b_{0,1}=1 \\
b_{i, 1}=1, i \geq 1 \\
b_{2,2}=\sigma_{\AA}^{2} \cdot \kappa_{1}^{2}
\end{array}\right\}
$$


and

$$
\underline{\tilde{r}} \triangleq \frac{\underline{r}}{\|\underline{x}\|}, \underline{\underline{r}} \triangleq \underline{r}_{x}
$$

These expressions will now be proven.

Proof: We will begin with the proof of (80) and the method of 2-step mathematical induction will be used to prove the desired expression. Note that (80) is valid for $i \in \mathcal{Z}, i \geq 1$.

$i=1$ and $i=2$ : If we apply formula (80) for $i=2$ and $i=3$ it is straightforward to show that it is verified.

$i=k-1, i=k$ : Let us assume that this formula is correct for $i=k-1$ and $i=k$, that is

$$
\begin{aligned}
& \underline{u}_{k-1}= \frac{j^{k-1}}{\sigma_{\mathrm{A}}^{k-1} \kappa_{1} \kappa_{2} \cdots \kappa_{k-2}} \text { A } \\
& \cdot\left[\left(\sum_{n=1}^{\left\lfloor\frac{k-2}{2}\right\rfloor+1}(-1)^{n-1} \cdot b_{k-2, n} \underline{\tilde{r}}^{k+1-2 n}\right) \odot \underline{\mathrm{a}}\right]
\end{aligned}
$$

and

$$
\begin{aligned}
\underline{u}_{k}=\frac{j^{k}}{\sigma_{\AA}^{k} \kappa_{1} \kappa_{2} \cdots \kappa_{k-1}} \text { A } & {\left[\left(\sum_{n=1}^{\left\lfloor\frac{k-1}{2}\right\rfloor+1}(-1)^{n-1} \cdot b_{k-1, n} \underline{\tilde{r}}^{k+2-2 n}\right) \odot \underline{\mathrm{a}}\right] }
\end{aligned}
$$

$i=k+1$ : Based on the previous step one can show that

$$
\begin{aligned}
\underline{u}_{k+1}= & \frac{\underline{u}_{k}^{\prime}+\kappa_{k-1} \underline{u}_{k-1}}{\kappa_{k}} \\
= & \frac{j^{k+1}}{\sigma_{\AA}^{k+1} \kappa_{1} \kappa_{2} \cdots \kappa_{k}} \text { A } \\
& \times\left[\left(\sum_{n=1}^{\left.\left.\frac{\lfloor-1}{2}\right\rfloor\right]+1}(-1)^{n-1} b_{k-1, n} \underline{\tilde{r}}^{k+3-2 n}\right) \odot \underline{\mathrm{a}}\right] \\
& +\frac{j^{k+1} \sigma_{\AA}^{2} \kappa_{k-1}^{2}}{\sigma_{\AA}^{k+1} \kappa_{1} \kappa_{2} \cdots \kappa_{k}} \text { A } \\
& \cdot\left[\left(\sum_{n=2}^{\left\lfloor\frac{k}{2}\right\rfloor+1}(-1)^{n-1} b_{k-2, n-1} \underline{\tilde{r}}^{k+3-2 n}\right) \odot \underline{\mathrm{a}}\right] .
\end{aligned}
$$

The formula of (85) is not the same as the one we are trying to prove. From the first sum of the right hand side, the term for $n=\left\lfloor\frac{k}{2}\right\rfloor+1$ is missing, while from the second sum, the term for $n=1$ is missing. We will show now, that both these terms equal to 0 , or equivalently, that the corresponding coefficients $b_{i, n}=0$.

The coefficients $b_{i, n}$ are 0 if any of the following 3 conditions is satisfied: $i=n \wedge i>2$ OR $i<n$ OR $n=0$. Therefore, the second missing term can easily be proven to be equal to 0 , since $b_{k-2, n-1} \stackrel{n \equiv 1}{=} b_{k-2,0}=0$. For the first term, observe that if $k=2 \rho+1, \rho=1,2 \ldots$, then $\left\lfloor\frac{k}{2}\right\rfloor+1=\rho+1=\left\lfloor\frac{k-1}{2}\right\rfloor+1$.

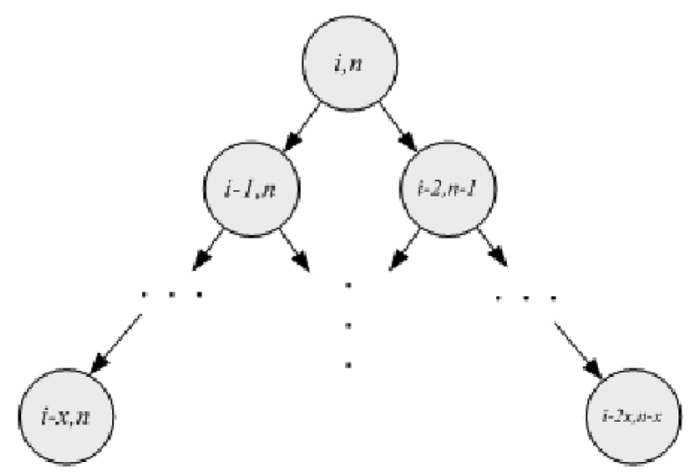

Fig. 4. Graphical representation of the iterative generation of $b_{i, n}$.

Therefore, if $k$ is odd, the upper limit of the first sum is, in fact equal to $\left\lfloor\frac{k}{2}\right\rfloor+1$, as it should be. If $k=2 \rho, \rho=1,2 \ldots$ then the first term is

$$
n=\left\lfloor\frac{k}{2}\right\rfloor+1=\rho+1 .
$$

Therefore, we have to show that $b_{2 \rho-1, \rho+1}=0$.

The coefficients $b_{i, n}$ can be defined recursively, as can be seen in (82). In every step of the recursion, the coefficient can be expressed as the sum of two terms, in each one of which there is another coefficient, with different indices. In every step, in both of the emerging coefficients, the difference between the first and the second index is decreased by one. However, the indexes of the second coefficient decrease faster. In order for $b_{2 \rho-1, \rho+1}=$ 0 to be zero, all of the emerging coefficients in this recursion must be also zero. Equivalently, the indexes of all the emerging coefficients must satisfy one of the conditions mentioned above. We can represent this recursion as a binary tree, see Fig. 4, each node of which will represent a different coefficient. For the root of this tree to be 0 , all the leafs have to be zero too. However, if we prove that the coefficient emerging always from the second term of the recursive (82), that is the rightmost leaf of the tree is equal to 0 then, all the other coefficients will be equal to 0 as well, because even though the difference in the indexes is decreased by the same amount in each recursion (each level of the tree), the indexes of the rightmost coefficients (the nodes in the path from the root to the rightmost coefficient) approach the limit $i=2$ faster than the nodes in any other path.

After $x$ steps of the recursion, the indexes of the rightmost leaf will be

$$
b_{i_{x}, n_{x}}=b_{2 \rho-1-2 x, \rho+1-x} .
$$

The coefficients will be zero if the indexes are equal, so $x=$ $\rho-2$. Therefore, after $x=\rho-2$ steps of the recursion, the indexes will be

$i_{x}=2 \rho-1-2(\rho-2)=3>2, \quad n_{x}=\rho+1-(\rho-2)=3>2$.

Thus, it has been proven, that all the coefficients for this term are 0 and the proof for the coordinate vectors is complete. The proof for the curvatures follows from this result and (81). 


\section{B. Proof of Theorem 1}

Proof: The method of mathematical induction will be used again. During this proof, variables with a superscript $\mathcal{H}_{c}$ will refer to the extended manifold curves, while variables with no superscript will refer to the coefficients and curvatures of the conventional array curves.

Based on (81), the calculation of the $i$ th curvature $\kappa_{i}$ requires the knowledge of all the coefficients $b_{k, n}, k=1, \ldots, i$, $n=1, \ldots,\left\lfloor\frac{i}{2}\right\rfloor$, whereas the calculation of the coefficients $b_{i, n}$, $n=1, \ldots,\left\lfloor\frac{i}{2}\right\rfloor$ require the knowledge of the curvature $\kappa_{i-1}$ and the that of some of the previous coefficients. The calculation of each coefficient is a function of the coefficients which precede it in the previous arrangement plus the curvature of the previous order. Therefore, if it is known that up to an order $i$

$$
\begin{aligned}
b_{k, n}^{\mathcal{H}_{c}} & =b_{k, n}, \quad k=1, \ldots, i, n=1, \ldots,\left\lfloor\frac{i}{2}\right\rfloor \\
\kappa_{k}^{\mathcal{H}_{c}} & =\frac{\kappa_{k}}{\sigma_{\text {A }}}, \quad k=1, \ldots,\left\lfloor\frac{i}{2}\right\rfloor
\end{aligned}
$$

then for the next order $i+1$, the coefficients of the array manifold and the extended array manifold will be equal as well. Since (82) is a two step recursion, we have to prove first that the necessary conditions hold for $i=1$ and $i=2$.

For $i=1$, it is

$$
\underline{b}_{1}^{\mathcal{H}_{c}}=[1]=\underline{b}_{1}, \quad \kappa_{1}^{\mathcal{H}_{c}}=\frac{\left\|\underline{\tilde{r}}^{2}\right\|}{\sigma_{\text {A }}}=\frac{\kappa_{1}}{\sigma_{\text {A }}} .
$$

For $i=2$, it is

$$
\begin{aligned}
b_{2,2}^{\mathcal{H}_{c}} & =\sigma_{\text {A }}^{2}\left(\kappa_{1}^{\mathcal{H}_{c}}\right)^{2}=\kappa_{1}^{2}=b_{2,2} \\
\kappa_{2}^{\mathcal{H}_{c}} & =\frac{1}{\sigma_{\text {A }}^{2} \kappa_{1}^{\mathcal{H}_{c}}}\left\|\tilde{r}^{3}-\sigma_{\AA}^{2}\left(\kappa_{1}^{\mathcal{H}_{c}}\right)^{2} \underline{\underline{r}}\right\|=\frac{\kappa_{2}}{\sigma_{\mathrm{A}}} .
\end{aligned}
$$

Now, it has to be proven that, if the conditions of (86) hold and given the previous result for the coefficients, the curvatures relation of (86) will hold for the next order $i+1$ as well. For the next order, $i+1$, based on (86), the coefficients $\underline{b}_{i+1}^{\mathcal{H}_{c}}$ and $\underline{b}_{i+1}$ will be equal. Thus, from (81) it is straightforward to show that

$$
\kappa_{i+1}^{\mathcal{H}_{c}}=\frac{\kappa_{i+1}}{\sigma_{\text {A }}}
$$

Now that the curvatures relation has been proven, by substituting (87) into (80) it is straightforward to show that (80) is also verified.

\section{APPENDIX B \\ DERIVATION OF CHRISTOFFEL MATRICES}

\section{A. Derivation of Equation (74)}

Based on the definitions of the Christoffel matrix of the first kind

$$
\Gamma_{1 \zeta, \mathcal{H}}=\operatorname{Re}\left\{\mathbb{\varpi}_{\mathcal{H}}^{H} \dot{\mathbb{T}}_{\zeta, \mathcal{H}}\right\}
$$

However $\dot{\mathbb{T}}_{\zeta, \mathcal{H}}=\dot{\mathrm{A}}_{p q} \rrbracket_{2} \otimes \underline{\dot{\mathrm{a}}}_{\zeta}+\ddot{\mathrm{A}}_{p q, \zeta} \rrbracket_{2} \otimes \underline{\mathrm{a}}+\dot{\mathrm{A}}_{\zeta} \mathbb{\mathrm { T }}+\mathrm{A} \dot{\mathbb{T}}_{\zeta}$ where $\ddot{\mathrm{A}}_{p q, \zeta} \triangleq \frac{\partial \dot{\mathrm{A}}_{p q}}{\partial \zeta}$ and $\dot{\mathbb{T}}_{\zeta} \triangleq \frac{\partial \mathbb{T}}{\partial \zeta}$. Thus

$$
\begin{aligned}
& \operatorname{Re}\left\{\mathbb{\varpi}_{\mathcal{H}}^{H} \dot{\mathbb{T}}_{\zeta, \mathcal{H}}\right\}
\end{aligned}
$$

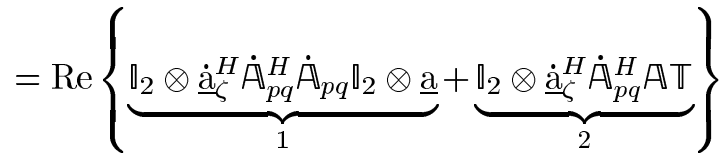

$$
\begin{aligned}
& +\operatorname{Re}\{\underbrace{\beth_{2} \otimes \underline{\mathrm{a}}^{H} \ddot{\mathrm{A}}_{p q, \zeta}^{H} \dot{\mathrm{A}} p q \rrbracket_{2} \otimes \underline{\mathrm{a}}}_{3}+\underbrace{\mathbb{I}_{2} \otimes \underline{\mathrm{a}}^{H} \ddot{\mathrm{A}}_{p q, \zeta}^{H} \mathrm{AT}}_{4}\} \\
& +\operatorname{Re}\{+\underbrace{\mathbb{\nabla}^{H} \dot{\mathrm{A}}_{\zeta}^{H} \dot{\mathrm{A}_{p q} \mathbb{D}_{2} \otimes \underline{\mathrm{a}}}}_{5}+\underbrace{\mathbb{\nabla}^{H} \dot{\mathrm{A}}_{\zeta}^{H} \mathrm{AT}}_{6}\} \\
& +\operatorname{Re}\{\underbrace{\dot{\mathbb{T}}_{\zeta}^{H} \mathbb{A}^{H} \dot{A}_{p q} \mathbb{\Xi}_{2} \otimes \underline{\mathrm{a}}}_{7}+\underbrace{\dot{\mathbb{V}}_{\zeta}^{H} A^{H} A \mathbb{T}}_{8}\} .
\end{aligned}
$$

Terms 1,4 , and 5 are all equal to $\mathbb{O}_{2}$ since they involve the inner products $\underline{\dot{\mathrm{a}}}_{\zeta}^{H} \underline{\mathrm{a}}=0$.

Term 2 .

$$
\begin{aligned}
& \operatorname{Re}\left\{\mathbb{I}_{2} \otimes \underline{\dot{\mathrm{a}}}^{H} \dot{\mathrm{A}}_{p q}^{H} \mathrm{~A} \mathbb{\mathrm { T }}\right\} \\
& =\operatorname{Re}\left\{\left[\begin{array}{ll}
\underline{\dot{\mathrm{a}}}_{\zeta}^{H} \dot{\mathrm{A}}_{p}^{H} \mathrm{~A} \underline{\dot{a}}_{p} & \dot{\mathbf{\mathrm { a }}}_{\zeta}^{H} \dot{\mathrm{A}}_{p}^{H} \text { A } \dot{\dot{a}}_{q} \\
\dot{\mathrm{a}}_{\zeta}^{H} \dot{\mathrm{A}}_{q}^{H} \mathrm{~A} \underline{\dot{a}}_{p} & \underline{\dot{a}}_{\zeta}^{H} \dot{\mathrm{A}}_{q}^{H} \mathrm{~A} \underline{\dot{a}}_{q}
\end{array}\right]\right\} \\
& =\operatorname{Re}\left\{\left[\begin{array}{ll}
\underline{\underline{\mathrm{a}}}_{\zeta}^{H} \underline{\dot{\mathrm{a}}}_{p} & \underline{\dot{\mathrm{a}}}_{\zeta}^{H} \underline{\dot{\mathrm{a}}}_{q} \\
\dot{\dot{\mathrm{a}}}_{\zeta}^{H} \underline{\dot{\mathrm{a}}}_{p} & \underline{\dot{\mathrm{a}}}_{\zeta}^{H} \underline{\dot{\mathrm{a}}}_{q}
\end{array}\right] \odot\left[\begin{array}{ll}
\underline{\dot{z}}_{p}^{H} \underline{z} & \underline{\dot{z}}_{p}^{H} \underline{z} \\
\dot{\dot{z}}_{q}^{H} \underline{z} & \underline{\dot{z}}_{q}^{H} \underline{z}
\end{array}\right]\right\} \\
& =\operatorname{Re}\left\{\mathbb{T}_{\underline{z}}^{H}{\underline{z \dot{a}_{\zeta}^{H}}}^{H} \mathbb{T}\right\} \text {. }
\end{aligned}
$$

Term 3:

$$
\begin{aligned}
\operatorname{Re} & \left\{\rrbracket_{2} \otimes \underline{\mathrm{a}}^{H} \ddot{\mathrm{A}}_{p q, \zeta}^{H} \dot{\mathrm{A}}_{p q}^{\rrbracket_{2}} \otimes \underline{\mathrm{a}}\right\} \\
& =\operatorname{Re}\left\{\left[\begin{array}{ll}
\underline{\mathrm{a}}^{H} \ddot{\mathrm{A}}_{p \zeta}^{H} \dot{\mathrm{A}}_{p} \underline{\mathrm{a}} & \underline{\mathrm{a}}^{H} \ddot{\mathrm{A}}_{p \zeta}^{H} \dot{\mathrm{A}}_{q} \underline{\mathrm{a}} \\
\underline{\mathrm{a}}^{H} \ddot{\mathrm{A}}_{q \zeta}^{H} \dot{\mathrm{A}}_{p} \underline{\mathrm{a}}^{H} \underline{\mathrm{a}}^{H} \ddot{\mathrm{A}}_{q \zeta}^{H} \dot{\mathrm{A}}_{q} \underline{\mathrm{a}}
\end{array}\right]\right\} \\
& =N \operatorname{Re}\left\{\left[\begin{array}{l}
\ddot{\ddot{z}}_{p \zeta}^{H} \\
\ddot{z}_{p \zeta}^{H}
\end{array}\right]\left[\begin{array}{ll}
\underline{\dot{z}}_{p} & \underline{\dot{z}}_{q}
\end{array}\right]\right\} N \operatorname{Re}\left\{\dot{\mathbb{\nabla}}_{\underline{z}, \zeta}^{H} \mathbb{\mathbb { T }}_{\underline{z}}\right\} .
\end{aligned}
$$

Term 6:

$$
\begin{aligned}
& \operatorname{Re}\left\{\mathbb{\eta}_{2} \otimes \underline{\dot{a}}^{H} \dot{\mathrm{A}}_{p q}^{H} \mathrm{AT}\right\} \\
& =\operatorname{Re}\left\{\left[\begin{array}{ll}
\dot{\underline{\mathrm{a}}}_{p}^{H} \dot{\mathrm{A}}_{\zeta}^{H} \mathrm{~A} \underline{\dot{\mathrm{a}}}_{p} & \dot{\mathrm{a}}_{p}^{H} \dot{\mathrm{A}} \dot{\mathrm{a}}_{\zeta}^{H} \mathrm{Al} \underline{\dot{\mathrm{a}}}_{q} \\
\dot{\mathrm{a}}_{q}^{H} \dot{\mathrm{A}}_{\zeta}^{H} \mathrm{~A} \underline{\dot{a}}_{p} & \underline{\dot{\mathrm{a}}}_{q}^{H} \dot{\mathrm{A}}_{\zeta}^{H} \mathrm{~A} \underline{\dot{a}}_{q}
\end{array}\right]\right\}
\end{aligned}
$$

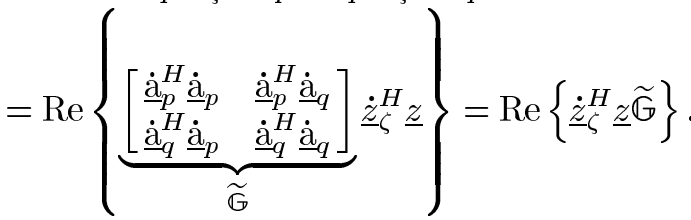

\section{Term 7:}

$$
\begin{aligned}
& \operatorname{Re}\left\{\dot{\mathbb{T}}_{\zeta}^{H} \mathrm{~A}^{H} \dot{\mathrm{A}}_{p q} \mathbb{1}_{2} \otimes \underline{\mathrm{a}}\right\}
\end{aligned}
$$

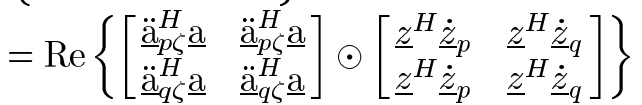

$$
\begin{aligned}
& =\operatorname{Re}\left\{\dot{\mathbb{T}}_{\zeta}^{H} \underline{\mathrm{a} z}^{H} \mathbb{T}_{\underline{z}}\right\} \text {. }
\end{aligned}
$$

Term 8:

$$
\operatorname{Re}\left\{\dot{\mathbb{T}}_{\zeta}^{H} \mathbb{A}^{H} \mathrm{~A} \mathbb{T}\right\}=\sigma_{\AA}^{2} \operatorname{Re}\left\{\dot{\mathbb{T}}_{\zeta}^{H} \mathbb{T}\right\}=\sigma_{\mathrm{A}}^{2} \boldsymbol{\Gamma}_{1 \zeta} \cdot
$$




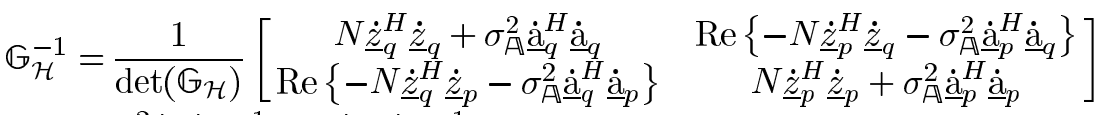

$$
\begin{aligned}
& =\frac{\sigma_{\AA}^{2}|\mathbb{G}| \mathbb{G}^{-1}+N\left|\mathbb{G}_{\underline{z}}\right| \mathbb{G}_{\underline{z}}^{-1}}{\operatorname{det}\left(\mathbb{G}_{\mathcal{H}}\right)}
\end{aligned}
$$

However

$$
\begin{aligned}
& \operatorname{det}\left(\mathbb{G}_{\mathcal{H}}\right)=N^{2} \underbrace{\left(\underline{\dot{z}}_{p}^{H} \underline{\dot{z}}_{p} \dot{\dot{z}}_{q}^{H} \underline{\dot{z}}_{q}-\operatorname{Re}\left\{\underline{\dot{z}}_{p}^{H} \dot{\dot{z}}_{q}\right\} \operatorname{Re}\left\{\underline{\dot{z}}_{q}^{H} \underline{\dot{\dot{z}}}_{p}\right\}\right)}_{\operatorname{det}\left(\mathbb{G}_{\underline{z}}\right)} \\
& +\sigma_{\AA}^{4} \underbrace{\left(\dot{\mathrm{a}}_{p}^{H} \dot{\underline{\mathrm{a}}}_{p} \dot{\underline{\mathrm{a}}}_{q}^{H} \dot{\underline{\mathrm{a}}}_{q}-\operatorname{Re}\left\{\dot{\mathrm{a}}_{p}^{H} \dot{\mathrm{a}}_{q}\right\} \operatorname{Re}\left\{\dot{\mathrm{a}}_{q}^{H} \dot{\underline{\mathrm{a}}}_{p}\right\}\right)}_{\operatorname{det}(\mathbb{G})}
\end{aligned}
$$

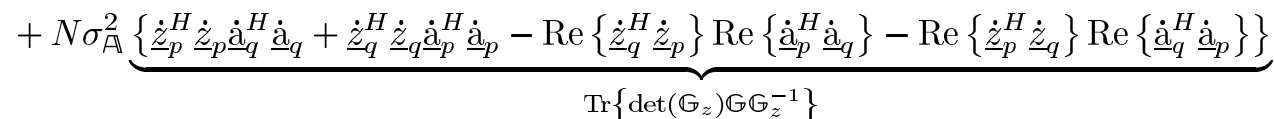

$$
\begin{aligned}
& =N^{2} \operatorname{det}\left(\mathbb{G}_{\underline{z}}\right)+\sigma_{\text {A }}^{4} \operatorname{det}(\mathbb{G})+N \sigma_{\AA}^{2} \operatorname{Tr}\left\{\operatorname{det}\left(\mathbb{G}_{\underline{z}}\right) \mathbb{G}_{\underline{\underline{z}}}^{-1}\right\}
\end{aligned}
$$

\section{B. Derivation of Equation (79)}

See (95), shown at the top of the page. However, [see (96), shown at the top of the page].

\section{REFERENCES}

[1] K. Fritzsche and H. Grauert, From Holomorphic Functions to Complex Manifolds, ser. Graduate Text in Mathematics. New York: Springer, 2002.

[2] A. Sleiman and A. Manikas, "Antenna array manifold: A simplified representation," in Proc. IEEE Int. Conf. Acoust., Speech, Signal Process. (ICASSP), 2000, vol. 5, pp. 3164-3167.

[3] A. Manikas, Differential Geometry in Array Processing. London, U.K.: Imperial College Press, 2004.

[4] I. Dacos and A. Manikas, "Estimating the manifold parameters of one-Dimensional array of sensors," J. Franklin Inst. (Eng. Appl. Math.), vol. 332B, no. 3, pp. 307-332, 1995.

[5] U. Guggenheimer, Differential Geometry. New York: McGraw-Hill, 1963.

[6] G. Efstathopoulos, G. Elissaios, and A. Manikas, "Geometric properties of 3-D array systems," WSEAS Trans. Commun., vol. 5, no. 10, pp. 1959-1964, 2006

[7] U. Lipschutz, Theory and Problems of Differential Geometry, ser. Shaum's Outline. New York: McGraw-Hill, 1969.

[8] A. Manikas, A. Sleiman, and I. Dacos, "Manifold studies of nonlinear antenna array geometries," IEEE Trans. Signal Process., vol. 49, no. 3, pp. 497-506, Mar. 2001.

[9] A. Manikas and C. Proukakis, "Modeling and estimation of ambiguities in linear arrays," IEEE Trans. Signal Process., vol. 46, no. 8, pp. 2166-2179, Aug. 1998.

[10] A. Manikas, H. R. Karimi, and I. Dacos, "Study of the detection and resolution capabilities of a one-dimensional array of sensors by using differential geometry," Proc. Inst. Electr. Eng.-Radar, Sonar, Navig., vol. 141, no. 2, pp. 83-92, 1994.

[11] A.-K. Kleinsteuber and M. Seghouane, "On the deterministic CRB for DOA estimation in unknown noise fields using sparse sensor arrays," IEEE Trans. Signal Process., vol. 56, no. 2, pp. 860-864, Feb. 2008.

[12] N. J. Malloy, "Array manifold geometry and sparse volumetric array design optimization," in Confe. Rec. 41st Asilomar Conf. Signals, Syst., Comput., Nov. 2007, pp. 1257-1261.

[13] M. Buhren, M. Pesavento, and J. Bohme, "Virtual array design for array interpolation using differential geometry," in Proc. IEEE Int. Conf. Acoust., Speech, Signal Process. (ICASSP), May 2004, vol. 2, pp. ii-229-ii-232.
[14] U. Baysal and R. L. Moses, "On the geometry of isotropic arrays," IEEE Trans. Signal Process., vol. 51, no. 6, pp. 1469-1478, Jun. 2003.

[15] H. Gazzah and K. Abed-Meraim, "Optimum ambiguity-free directional and omnidirectional planar antenna arrays for DOA estimation," IEEE Trans. Signal Process., vol. 57, no. 10, pp. 3942-3953, Oct. 2009.

[16] D. Asztely, B. Ottersten, and A. Swindlehurst, "Generalised array manifold model for wireless communication channels with local scattering," Proc. Inst. Electr. Eng._Radar, Sonar, Navig., vol. 145, no. 1, pp. 51-57, Feb. 1998

[17] M. Hawkes and A. Nehorai, "Acoustic vector-sensor beamforming and capon direction estimation," IEEE Trans. Signal Process., vol. 46, no. 9, pp. 2291-2304, Sep. 1998.

[18] M. A. Doron and E. Doron, "Wavefield modeling and array processing. III. Resolution capacity," IEEE Trans. Signal Process., vol. 42, no. 10, pp. 2571-2580, 1994.

[19] M. A. Doron and E. Doron, "Wavefield modeling and array processing. II. Algorithms," IEEE Trans. Signal Process., vol. 42, no. 10, pp. 2560-2570, 1994.

[20] M. A. Doron and E. Doron, "Wavefield modeling and array processing. I. Spatial sampling," IEEE Trans. Signal Process., vol. 42, no. 10, pp. 2549-2559, 1994

[21] A. Manikas and L. K. Huang, "STAR channel estimation in DS-CDMA communication systems," Proc. Inst. Electr. Eng.-Commun., vol. 151, no. 4, pp. 387-393, 2004.

[22] D. J. Sadler and A. Manikas, "Blind reception of multicarrier DS-CDMA using antenna arrays," IEEE Trans. Wireless Commun., vol. 2 , no. 6, pp. 1231-1239, 2003.

[23] A. Manikas and J. W. P. Ng, "Crossed-dipole arrays for asynchronous DS-CDMA systems," IEEE Trans. Antennas Propag., vol. 52, no. 1, pp. 122-131, 2004.

[24] B. Friedlander and A. J. Weiss, "Performance of diversely polarized antenna arrays for correlated signals," IEEE Trans. Aerosp. Electron. Syst., vol. 28, no. 3, pp. 869-879, 1992.

[25] J. Li and J. R. T. Compton, "Angle and polarization estimation in a coherent signal environment," IEEE Trans. Aerosp. Electron. Syst., vol. 29, no. 3, pp. 706-716, 1993.

[26] J. W. P. Ng and A. Manikas, "Diversely polarised arrays in DS-CDMA: A space-time channel estimation approach," in Proc. IEEE Int. Conf. Acoust., Speech, Signal Process. (ICASSP), 2002, vol. 3, p. III-2617.

[27] J. W. P. Ng and A. Manikas, "MIMO array DS-CDMA system: A blind space-time-Doppler estimation/reception," in Proc. IEEE Int. Conf. Acoust., Speech, Signal Process. (ICASSP), 2004, vol. 2, pp. 337-340.

[28] A. Manikas, A. Alexiou, and H. R. Karimi, "Comparison of the ultimate direction-finding capabilities of a number of planar array geometries," Proc. Inst. Electr. Eng.—Radar, Sonar, Navig., vol. 144, no. 6, pp. 321-329, 1997. 
[29] N. Dowlut and A. Manikas, "A polynomial rooting approach to superresolution array design," IEEE Trans. Signal Process., vol. 48, no. 6 , pp. 1559-1569, Jun. 2000.

[30] G. Efstathopoulos and A. Manikas, "Investigation of the spatio-temporal array manifold of linear arrays using differential geometry," in Proc. 8th Int. Conf. Signal Process., 2006, vol. 1.

[31] P. Stoica and A. Nehorai, "Music, maximum likelihood, and Cramer-Rao bound," IEEE Trans. Acoust., Speech, Signal Process., vol. 37 , no. 5 , pp. $720-741,1989$.

[32] T. Willmore, An Introduction to Differential Geometry. London, U.K.: Oxford Univ. Press, 1959.

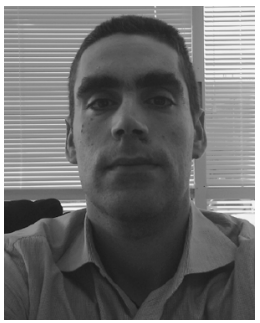

Georgios Efstathopoulos (M'07) received the Diploma (with Hons.) degree in electrical and computer engineering from the National Technical University of Athens, Greece, in 2004 and the Ph.D. degree in array communications and signal processing from Imperial College London in 2008, under the supervision of Prof. A. Manikas.

$\mathrm{He}$ joined the Communications and Signal Processing Group at Imperial College London in 2004. His research interests lie in the areas of array processing for communications, target detection and tracking, differential geometry, and array calibration.

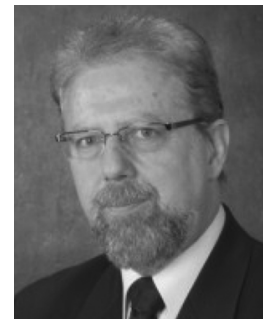

Athanassios Manikas (SM'02) holds the Chair of Communications and Array Processing in the Department of Electrical and Electronic Engineering, Imperial College London, and he is currently the Technical Chair/Lead of the University Defence Research Centre in Signal Processing (DSTL/EPSRC). He is leading a strong group of researchers at Imperial College and has supervised successfully 33 Ph.D.'s and more than 100 Master's project-students.

$\mathrm{He}$ is on the Editorial Board of the IET Proceedings Signal Processing and has held a number of research consultancies for the European Union, industry, and government organizations. Also, he has had various technical chairs at international conferences and has been a TPC member of major IEEE conferences. He has served as an Expert Witness in the High Court of Justice (U.K.) and as a member of the Royal Society's International Fellowship Committee (January 1, 2008 until December 31, 2010). He is a Fellow of IET (U.K.) and a Chartered Engineer. 